\title{
Review
}

\section{Green Synthesis and Potential Antibacterial Applications of Bioactive Silver Nanoparticles: A Review}

\author{
Md. Amdadul Huq ${ }^{1, *,+} \mathbb{D}$, Md. Ashrafudoulla ${ }^{2,+}$, M. Mizanur Rahman ${ }^{3} \mathbb{D}$, Sri Renukadevi Balusamy ${ }^{4, * \mathbb{D}}$ \\ and Shahina Akter $5, *$
}

check for

updates

Citation: Huq, M.A.;

Ashrafudoulla, M.; Rahman, M.M.; Balusamy, S.R.; Akter, S. Green

Synthesis and Potential Antibacterial Applications of Bioactive Silver Nanoparticles: A Review. Polymers 2022, 14, 742. https://doi.org/ $10.3390 /$ polym 14040742

Academic Editor: Zhen Zhang

Received: 20 January 2022

Accepted: 12 February 2022

Published: 15 February 2022

Publisher's Note: MDPI stays neutral with regard to jurisdictional claims in published maps and institutional affiliations.

Copyright: (c) 2022 by the authors Licensee MDPI, Basel, Switzerland. This article is an open access article distributed under the terms and conditions of the Creative Commons Attribution (CC BY) license (https:// creativecommons.org/licenses/by/ $4.0 /$ )
1 Department of Food and Nutrition, College of Biotechnology and Natural Resource, Chung-Ang University, Anseong 17546, Korea

2 Department of Food Science and Technology, Chung-Ang University, Anseong 17546, Korea; ashrafmiu584@gmail.com

3 Department of Biotechnology and Genetic Engineering, Faculty of Biological Science, Islamic University, Kushtia 7003, Bangladesh; rahmanmm@btge.iu.ac.bd

4 Department of Food Science and Biotechnology, Sejong University, Gwangjin-gu, Seoul 05006, Korea

5 Department of Food Science and Biotechnology, Gachon University, Seongnam 461701, Korea

* Correspondence: amdadbge@gmail.com or amdadbge100@cau.ac.kr (M.A.H.); renucoimbatore@gmail.com (S.R.B.); shahinabristy16@gmail.com (S.A.)

+ These authors contributed equally to this work.

\begin{abstract}
Green synthesis of silver nanoparticles (AgNPs) using biological resources is the most facile, economical, rapid, and environmentally friendly method that mitigates the drawbacks of chemical and physical methods. Various biological resources such as plants and their different parts, bacteria, fungi, algae, etc. could be utilized for the green synthesis of bioactive AgNPs. In recent years, several green approaches for non-toxic, rapid, and facile synthesis of AgNPs using biological resources have been reported. Plant extract contains various biomolecules, including flavonoids, terpenoids, alkaloids, phenolic compounds, and vitamins that act as reducing and capping agents during the biosynthesis process. Similarly, microorganisms produce different primary and secondary metabolites that play a crucial role as reducing and capping agents during synthesis. Biosynthesized AgNPs have gained significant attention from the researchers because of their potential applications in different fields of biomedical science. The widest application of AgNPs is their bactericidal activity. Due to the emergence of multidrug-resistant microorganisms, researchers are exploring the therapeutic abilities of AgNPs as potential antibacterial agents. Already, various reports have suggested that biosynthesized AgNPs have exhibited significant antibacterial action against numerous human pathogens. Because of their small size and large surface area, AgNPs have the ability to easily penetrate bacterial cell walls, damage cell membranes, produce reactive oxygen species, and interfere with DNA replication as well as protein synthesis, and result in cell death. This paper provides an overview of the green, facile, and rapid synthesis of AgNPs using biological resources and antibacterial use of biosynthesized AgNPs, highlighting their antibacterial mechanisms.
\end{abstract}

Keywords: green synthesis; silver nanoparticles; antibacterial application; antibacterial mechanisms

\section{Introduction}

Nanotechnology is an emerging field of research, with numerous applications in science and technology, especially in the development of different nanomaterials and nanoparticles. Nanoparticles (NPs) are small particles of size from $1 \mathrm{~nm}$ to $100 \mathrm{~nm}$ and have gained significant interest from scientists due to their multiple applications in diverse fields of science such as biomedicine, agriculture, pharmaceutics, textile, food technology, catalysis, sensors, mechanics, electronics, and optics [1,2]. There are different varieties of nanoparticles, including silver, gold, zinc, cadmium sulfide, copper, iron, titanium dioxide, etc., with unique characteristics [2-5]. Among different nanoparticles, silver nanoparticles 
(AgNPs) have been one of the most popular subjects of study in recent decades due to their wide scope of application in different branches of biomedical science as antibacterial, antifungal, antioxidant, anti-cancer, anti-inflammatory, drug delivery, wound dressings, biosensors, and biocatalysis, etc. [6-12]. Some recent studies have shown the strong antimicrobial, antioxidant, and anti-cancer activities of green synthesized AgNPs [6-8]. The biosynthesized AgNPs were also effectively used to degrade various toxic chemicals [9]. Moreover, green synthesized AgNPs have many other applications in different branches of biotechnology such as water filtration, sanitization, food preservation, production of cosmetics, nano-insecticides, and nanopesticides, etc. [10,11,13]. Green synthesized AgNPs have been reported as potential antibacterial agents against various Gram-positive and Gramnegative pathogenic bacteria, including Salmonella epidermidis, Salmonella Typhimurium, Pseudomonas aeruginosa, Staphylococcus aureus, Streptococcus pyogens, Escherichia coli, Bacillus subtilis, Vibrio parahaemolyticus, Streptococcus pneumoniae, Enterobacter hormaechei, Salmonella paratyphi, Klebsiella pneumoniae, Aeromonas hydrophila, Pseudomonas fluorescens, Flavobacterium branchiophilum, Enterobacter aerogenes, Shigella flexneri, Xanthomonas axonopodis, Salmonella enterica, etc. $[2,3,6,11-13]$.

Various physical and chemical methods such as physiochemical [14], electrochemical [15], photochemical [16], chemical reduction [17], and microwave irradiation [18] are commonly used for the synthesis of these nanoparticles. The main drawbacks of these methods are that they are expensive and hazardous because of the usage of toxic ingredients, costly, demand labor-intensive equipment and the generation of hazardous byproducts $[2,5,19]$. Due to the various drawbacks of physicochemical methods, researchers are currently focusing more on biological approaches for eco-friendly, non-toxic, inexpensive, and facile synthesis of nanoparticles (Figure 1). Green synthesis is an efficient process that uses natural compounds as reducing, capping, and stabilizing agents instead of expensive toxic chemicals. Various biological resources such as plants and their different parts (roots, leaves and fruit, etc.), bacteria, fungi, algae, etc. could be utilized for the green synthesis of bioactive nanoparticles [20-23]. Recently, green synthesis of AgNPs using plant extracts or microbes and their antimicrobial activity were widely investigated.

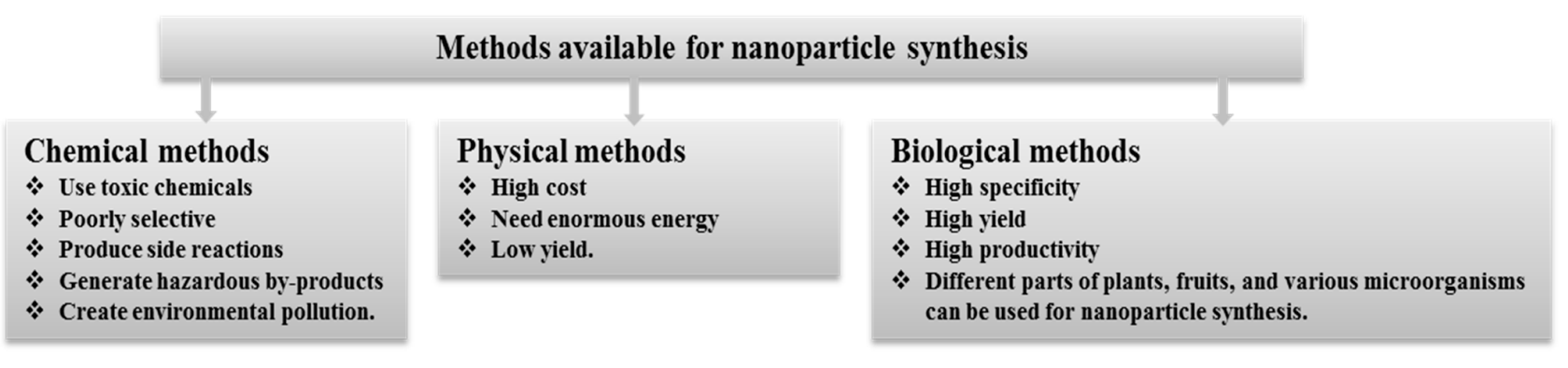

Figure 1. Different methods of nanoparticle synthesis.

Multidrug-resistant microorganisms are a serious threat to public health worldwide as different life-threatening infectious diseases are caused by these pathogens. There is a continuous increase in the number of multidrug-resistant bacterial strains due to mutation, pollution, changing environmental conditions and excessive use of drugs. To overcome this problem, scientists are trying to develop new drugs for the treatment of such microbial infections. Green synthesized AgNPs have been found to be effective for controlling these multidrug-resistant bacterial strains. This review provides an overview of green synthesis of AgNPs using different biological resources, various parameters essential for stable, easy and high yields, antibacterial applications and mechanisms of biosynthesized AgNPs as well as describing the prospect for their future development and potential antibacterial applications. 


\section{Green Synthesis of AgNPs}

Green synthesis of AgNPs using different biological agents such as plants, bacteria, fungi, algae and yeast is an economical, facile, and eco-friendly approach without generating any toxic byproducts. In recent years, both microbes and plants were extensively investigated for the green synthesis of AgNPs. Figure 2 illustrates the various steps of green synthesis of AgNPs using plants and microbes.

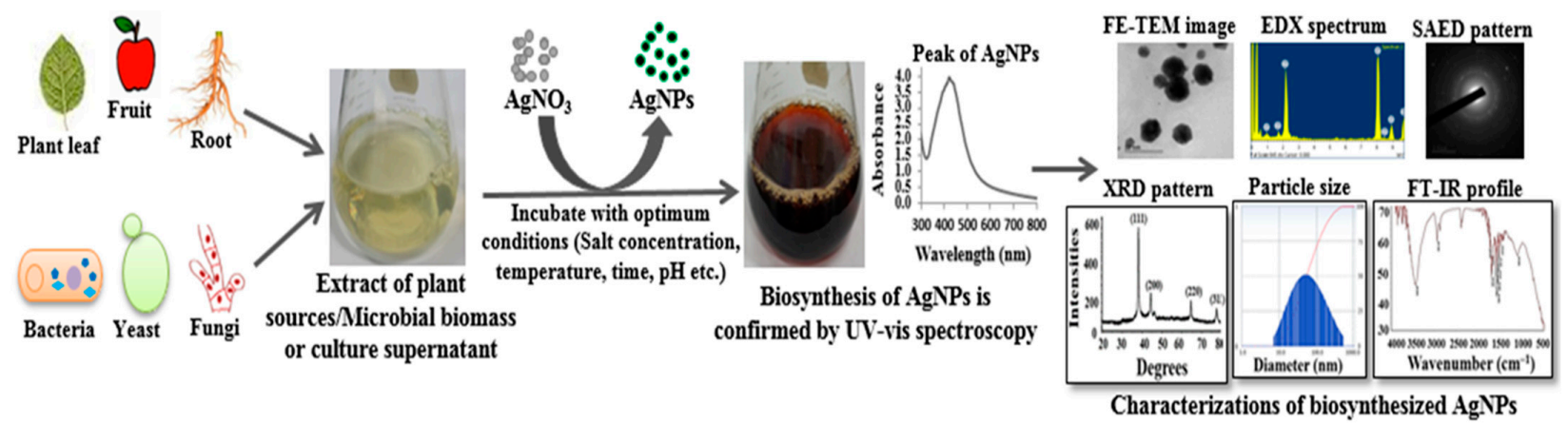

Figure 2. Schematic illustration of green synthesis and characterizations of AgNPs.

\section{Plant Mediated Synthesis of AgNPs}

Plant-mediated synthesis of AgNPs is a widely adopted technique due to the availability of various plants and their easy and safe utilization. Different parts of the plant including fruits, roots, flowers, leaves, peels, etc., have been successfully utilized for the green synthesis of bioactive AgNPs (Table 1). Plant extracts contain numerous bioactive compounds such as alkaloids, flavonoids, terpenoids, tannins, saccharides, phenols, vitamins, as well as various enzymes, amino acids, and proteins $[21,24,25]$. Due to the presence of these active biomolecules in plant extracts, synthesis of bioactive AgNPs using plants is more stable and easier. In the last few years, many studies have been conducted for the green synthesis of bioactive AgNPs using different parts of plants such as fruits, seeds, roots, flowers, stems, leaves, peels, etc. For instance, the leaf extract of Clerodendrum viscosum was used for facile, rapid, and eco-friendly synthesis of bioactive AgNPs [26]. They also investigated the antimicrobial efficacy of biosynthesized AgNPs against various pathogenic bacteria. Pawar and Patil [27] synthesized AgNPs using tuber extract of Eulophia herbacea. Fruit extract of Amomum villosum was used by Soshnikova et al. [28] for the facile synthesis of AgNPs. The seeds and roots of Durio zibethinus and Rheum palmatum, respectively, were used for green synthesis of AgNPs [29,30]. Peel extracts of different vegetables such as Lagenaria siceraria, Luffa cylindrica, Solanum lycopersicum, Solanum melongena and Cucumis sativus were investigated for synthesis of bioactive AgNPs [31]. Synthesis time, size and shape of synthesized AgNPs and their bioactivity varies greatly depending on the plant or part of the plant which was used for synthesis. For example, AgNPs of 10 to $30 \mathrm{~nm}$ in size were synthesized using root extract of Panax ginseng by two hours' reaction [32]. On the other hand, AgNPs of 5 to $15 \mathrm{~nm}$ were synthesized using leaf extract of Panax ginseng within 45 mins of reaction [33]. According to Adeyemi et al. [34], the leaf extract of Spondias mombin produced rod- or triangular-shaped AgNPs. However, the plant extract of Prunus africana, and Camellia sinensis produced spherical-shaped AgNPs [35]. Various parameters such as the extract salt ratio, incubation time, incubation temperature, $\mathrm{pH}$, etc. also greatly affected the easy, rapid, high, and stable synthesis of AgNPs using plant extracts $[3,6]$. The probable mechanism of plant-mediated synthesis of AgNPs is the chemistry of reduction and oxidation. It has been proposed that the plant extract contains vitamins, amino acids, proteins, enzymes, organic acid, flavonoids, terpenoids, alkaloids, polyphenols, and polysaccharides, which have significant roles for the reduction of silver salts as well as serve as capping and stabilizing agents $[21,24,25]$. 
Table 1. Green synthesis of AgNPs using plants and their antibacterial applications.

\begin{tabular}{|c|c|c|c|c|c|c|}
\hline Plants & Used Parts & Size $(\mathrm{nm})$ & Shape & $\begin{array}{c}\text { Optimum Synthesis } \\
\text { Parameters }\end{array}$ & Target Pathogens & References \\
\hline Plantago major & Leaf extract & $10-20$ & Spherical & $1 \mathrm{mM}, 70^{\circ} \mathrm{C}, 60 \mathrm{~min}$ & $\begin{array}{l}\text { S. aureus, E. coli, } \\
\text { P. aeruginosa }\end{array}$ & {$[25]$} \\
\hline $\begin{array}{c}\text { Prunus africana, Camellia } \\
\text { sinensis }\end{array}$ & Plant extract & $10-19$ & Spherical & $0.5 \mathrm{mM}, 25^{\circ} \mathrm{C}, 24 \mathrm{~h}$ & E. coli, K. pneumoniae & {$[35]$} \\
\hline Tasmanian flax-lily & Dried leaves extract & Av. 70 & Spherical & $0.1 \mathrm{mM}, 60^{\circ} \mathrm{C}, 25 \mathrm{~min}$ & $\begin{array}{l}\text { S. aureus, S. epidermidis, } P \text {. } \\
\text { aeruginosa, C. albican }\end{array}$ & [36] \\
\hline Carduus crispus & Plant extract & $33-131$ & NA & $\begin{array}{c}1 \mathrm{mM} \text {, room } \\
\text { temperature, } 24 \mathrm{~h}\end{array}$ & E. coli, M. luteus & [37] \\
\hline $\begin{array}{l}\text { Anastatica hierochuntica, } \\
\text { Artemisia absinthium }\end{array}$ & $\begin{array}{l}\text { Plant and seed } \\
\text { extracts }\end{array}$ & $\begin{array}{l}\text { Av. 114, } \\
125.5\end{array}$ & Spherical & $\begin{array}{l}1 \mathrm{mM} \text {, room } \\
\text { temperature, } 48 \mathrm{~h}\end{array}$ & $\begin{array}{l}\text { P. aeruginosa, E. coli, } \\
\text { S. aureus, C. albicans }\end{array}$ & [38] \\
\hline Lantana trifolia & Leaf extract & $5-70$ & Spherical & $1.5 \mathrm{M}, 35^{\circ} \mathrm{C}, 2 \mathrm{~h}$ & $\begin{array}{l}\text { S. aureus, C. albicans, } \\
\text { E. coli, P. aeruginosa, } \\
\text { B. subtilis }\end{array}$ & [39] \\
\hline Blumea eriantha & Plant extract & $10-60$ & Spherical & $\begin{array}{c}1 \% \text {, ambient } \\
\text { temperature, } 24 \mathrm{~h}\end{array}$ & $\begin{array}{l}\text { S.aureus, B. subtilis, } \\
\text { B. cereus, E. coli }\end{array}$ & {$[40]$} \\
\hline Cucumis prophetarum & Leaf extract & $30-50$ & Polymorphic & $1 \mathrm{mM}, 80^{\circ} \mathrm{C}, 3 \mathrm{~h}$ & S. typhi, S. aureus & [41] \\
\hline Clerodendrum viscosum & Leaf extract & $36-74$ & Spherical & $1 \mathrm{mM}, 60{ }^{\circ} \mathrm{C}, 60 \mathrm{~min}$ & $\begin{array}{l}\text { E. coli, } P \text {. aeruginosa, } \\
\text { B. subtilis, S. aureus }\end{array}$ & {$[26]$} \\
\hline Grape & $\begin{array}{l}\text { Proanthocyanidin } \\
\text { from seed }\end{array}$ & $100-120$ & Aggregated & $\begin{array}{c}\text { Ambient temperature, } \\
2-3 \mathrm{~h}\end{array}$ & S. aureus, P. aeruginosa, E. coli & {$[42]$} \\
\hline Spondias mombin & Leaf extract & & Rod or triangular & $1 \mathrm{mM}$, room temperature & S. aureus, $P$. aeruginosa, E. coli & {$[34]$} \\
\hline Eulophia herbacea & Tuber extract & Av. 11.7 & NA & $\begin{array}{c}1 \mathrm{mM} \text {, room } \\
\text { temperature, } 5 \mathrm{~h}\end{array}$ & $\begin{array}{c}\text { E. coli, S. aureus, } \\
\text { P. aruginosa, B. subtilis }\end{array}$ & {$[27]$} \\
\hline Torreya nucifera & Leaf extract & $10-125$ & Spherical & $1 \mathrm{M}, 20^{\circ} \mathrm{C}, 24 \mathrm{~h}$ & S. typhimurium & [43] \\
\hline Chlorophytum borivilianum & Callus extracts & $35-168$ & Spherical & $\begin{array}{c}1 \mathrm{mM} \text {, room } \\
\text { temperature, } 5 \mathrm{~h}\end{array}$ & $\begin{array}{l}\text { B. subtilis, S. aureus, } \\
\text { P. aeruginosa, E. coli }\end{array}$ & {$[44]$} \\
\hline $\begin{array}{l}\text { Purple heart plant } \\
\text { Phoenix dactylifera }\end{array}$ & $\begin{array}{l}\text { Leaves extract } \\
\text { Root hair extract }\end{array}$ & $\begin{array}{l}\text { Av. } 104.6 \\
21-41\end{array}$ & $\begin{array}{c}\text { NA } \\
\text { Spherical }\end{array}$ & $\begin{array}{c}50 \mathrm{mM}, 65^{\circ} \mathrm{C} \\
0.1 \mathrm{mM}, 50^{\circ} \mathrm{C}, 48 \mathrm{~h}\end{array}$ & $\begin{array}{l}\text { E. coli, S. aureus } \\
\text { C. albicans, E. coli }\end{array}$ & $\begin{array}{l}{[45]} \\
{[46]}\end{array}$ \\
\hline Taraxacum officinale & Leaf extract & $5-30$ & Spherical & $\begin{array}{l}1 \mathrm{mM} \text {, room } \\
\text { temperature, } 15 \mathrm{~min}\end{array}$ & $\begin{array}{c}\text { X. axonopodis, } \\
\text { P. syringae } \\
\text { P. aeruginosa, }\end{array}$ & [47] \\
\hline Chicory & Seed exudates & $\leq 25$ & Spherical & $5 \mathrm{mM}, 30^{\circ} \mathrm{C}$ & $\begin{array}{l}\text { K. pneumoniae, } \\
\text { A. baumannii, F. solani } \\
\text { E. coli, S. epidermidis, }\end{array}$ & [48] \\
\hline Punica granatum & Peel extract & $20-40$ & Spherical & $\begin{array}{c}0.1 \mathrm{mM} \text {, room } \\
\text { temperature, } 72 \mathrm{~h}\end{array}$ & $\begin{array}{l}\text { P. aeruginosa, S. typhi, } \\
\text { P. vulgaris, S. aureus, } \\
\text { K. pneumonia }\end{array}$ & [49] \\
\hline Durio Zibethinus & Seed extract & $20-75$ & Spherical, rod & $\begin{array}{c}1.5 \mathrm{mM} \text {, in sunlight, } 30 \\
\mathrm{~min}\end{array}$ & $\begin{array}{c}\text { E. coli, B. subtilis, } \\
\text { S. typhimurium, S. typhi }\end{array}$ & [29] \\
\hline Market vegetable & $\begin{array}{c}\text { Vegetable waste } \\
\text { extract }\end{array}$ & $10-90$ & Spherical & $1 \mathrm{mM}, 37^{\circ} \mathrm{C}, 5 \mathrm{~h}$ & $\begin{array}{c}\text { Klebsiella sp., Staphylococcus } \\
\text { sp. }\end{array}$ & [50] \\
\hline Rheum palmatum & Root extract & $44-113$ & $\begin{array}{l}\text { Hexagonal, } \\
\text { spherical }\end{array}$ & $\begin{array}{c}2 \mathrm{mM} \text {, room } \\
\text { temperature, } 24 \mathrm{~h}\end{array}$ & S. aureus, $P$. aeruginosa & [30] \\
\hline Angelica pubescens & Root extract & $20-50$ & Quasi-spherical & $5 \mathrm{mM}, 80^{\circ} \mathrm{C}, 50 \mathrm{~min}$ & $\begin{array}{l}\text { S. aureus, } P \text {. aeruginosa, } \\
\text { E. coli, S. enterica }\end{array}$ & [51] \\
\hline Protium serratum & Leaf extract & Av. 74.5 & Spherical & $1 \mathrm{mM}, 25^{\circ} \mathrm{C}, 4 \mathrm{~h}$ & $\begin{array}{c}\text { P. aeruginosa, E. coli, } \\
\text { B. subtilis }\end{array}$ & [52] \\
\hline Amomum villosum & Dried fruit extract & $5-15$ & Spherical & $\begin{array}{c}1 \mathrm{mM} \text {, room } \\
\text { temperature, } 3 \mathrm{~s}\end{array}$ & S. aureus, E. coli & [28] \\
\hline Glycyrrhiza uralensis & Root extract & $5-15$ & Spherical & $1 \mathrm{mM}, 80^{\circ} \mathrm{C}, 40 \mathrm{~min}$ & $\begin{array}{c}\text { E. coli, S. aureus, } P \text {. aeruginosa, } \\
\text { S. enterica }\end{array}$ & [53] \\
\hline Ficus palmata & Leaf extract & $28-33$ & Spherical & $\begin{array}{c}2 \mathrm{mM} \text {, room } \\
\text { temperature, } 6 \mathrm{~h}\end{array}$ & $\begin{array}{c}\text { S. pneumonia, E. coli, } \\
\text { P. aeruginosa, } \\
\text { K. pneumonia, } \\
\text { P. vulgaris }\end{array}$ & [54] \\
\hline Euphorbia antiquorum & Latex extract & $10-50$ & Spherical & $\begin{array}{c}1 \mathrm{mM} \text {, room } \\
\text { temperature, } 24 \mathrm{~h}\end{array}$ & $\begin{array}{c}\text { K. Pneumoniae, } \\
\text { P. mirabilis, V. cholerae, } \\
\text { E. faecalis }\end{array}$ & [55] \\
\hline Ocimum Sanctum & Leaf extract & Av. 14.6 & Spherical & $2 \mathrm{mM}, 35^{\circ} \mathrm{C}, 4 \mathrm{~h}$ & E. coli & {$[56]$} \\
\hline Moringa stenopetala & Leaf extract & Av. 11.4 & NA & $1 \mathrm{mM}, 60^{\circ} \mathrm{C}, 15 \mathrm{~min}$ & $\begin{array}{l}\text { S. aureus, E. coli } \\
\text { P. aeruginosa, E. coli, }\end{array}$ & [57] \\
\hline Euphrasia officinalis & Leaf extract & Av. 40.3 & Quasi-spherical & $1 \mathrm{mM}, 65^{\circ} \mathrm{C}, 19 \mathrm{~min}$ & $\begin{array}{c}\text { S. aureus, } \\
\text { V. parahaemolyticus. }\end{array}$ & [58] \\
\hline Siberian ginseng & Dried stem & Av. 14.6 & Spherical & $1 \mathrm{mM}, 80^{\circ} \mathrm{C}, 1.5 \mathrm{~h}$ & $\begin{array}{c}\text { S. aureus, B. anthracis, } \\
\text { V. parahaemolyticus. } \\
\text { E. coli }\end{array}$ & [59] \\
\hline Borago officinalis & Leaf extract & $30-80$ & $\begin{array}{l}\text { Spherical, } \\
\text { hexagonal, } \\
\text { irregular }\end{array}$ & $1 \mathrm{mM}, 65^{\circ} \mathrm{C}, 68 \mathrm{~s}$ & $\begin{array}{l}\text { P. aeruginosa, E. coli, } \\
\text { V. parahaemolyticus, } \\
\text { S. aureus }\end{array}$ & {$[60]$} \\
\hline Cocoa pod & Husk extract & $4-32$ & Spherical & $\begin{array}{c}1 \mathrm{mM}, 30^{\circ} \mathrm{C}, \text { few } \\
\text { minutes }\end{array}$ & $\begin{array}{c}\text { E. coli, K. pneumoniae, } \\
\text { S. pyogenes, S. aureus, } \\
\text { P. aeruginosa }\end{array}$ & {$[61]$} \\
\hline $\begin{array}{c}\text { Lagenaria siceraria, Luffa } \\
\text { cylindrica, Solanum } \\
\text { lycopersicum, Solanum } \\
\text { melongena, Cucumis sativus }\end{array}$ & $\begin{array}{l}\text { Vegetable peel } \\
\text { extract }\end{array}$ & up to 20 & Spherical & $2 \mathrm{mM}, 80^{\circ} \mathrm{C}, 10 \mathrm{~min}$ & E. coli, K. pneumoniae & [31] \\
\hline Azadirachta indica & Leaf extract & Av. 34 & Spherical & $\begin{array}{c}1 \mathrm{mM} \text {, room } \\
\text { temperature, } 24 \mathrm{~h}\end{array}$ & S. aureus, E. coli & [62] \\
\hline
\end{tabular}


Table 1. Cont.

\begin{tabular}{|c|c|c|c|c|c|c|}
\hline Plants & Used Parts & Size $(\mathrm{nm})$ & Shape & $\begin{array}{c}\text { Optimum Synthesis } \\
\text { Parameters }\end{array}$ & Target Pathogens & References \\
\hline Pedalium murex & Leaf extract & $10-50$ & Spherical & $10 \mathrm{mM}, 20 \mathrm{~min}$ & $\begin{array}{l}\text { B. subtilis, S. aureus, } \\
\text { E. coli, M. flavus, } \\
\text { P. aeruginosa, B. pumilus, } \\
\text { K. pheumoniae }\end{array}$ & {$[63]$} \\
\hline Cassia fistula & Leaf extract & $40-50$ & Spherical & $\begin{array}{c}1 \mathrm{mM} \text {, room } \\
\text { temperature, overnight }\end{array}$ & $\begin{array}{c}\text { B. subtilis, S. aureus, } \\
\text { C. kruseii, } \\
\text { T. mentagrophytes }\end{array}$ & {$[64]$} \\
\hline Psidium guajava & Leaf extract & $10-90$ & Spherical & $1 \mathrm{mM}, 30^{\circ} \mathrm{C}, 10 \mathrm{~min}$ & P. aeruginosa & {$[65]$} \\
\hline Coffea arabica & $\begin{array}{l}\text { Seed } \\
\text { extract }\end{array}$ & $10-150$ & $\begin{array}{l}\text { Spherical, } \\
\text { ellipsoidal }\end{array}$ & $\begin{array}{c}20 \mathrm{mM} \text {, room } \\
\text { temperature, } 2 \mathrm{~h}\end{array}$ & E. coli, S. aureus & {$[66]$} \\
\hline Styrax benzoin & $\begin{array}{l}\text { Benzoin gum } \\
\text { extract }\end{array}$ & $12-38$ & Spherical & $1 \mathrm{mM}, 60^{\circ} \mathrm{C}, 5 \mathrm{~h}$ & $\begin{array}{c}\text { E. coli, P. aeruginosa, } \\
\text { S. aureus, C. tropicalis } \\
\text { P. vulgaris, }\end{array}$ & [21] \\
\hline Cardiospermum halicacabum & Leaf extract & Av. 23 & Cubic & $\begin{array}{c}1 \mathrm{mM} \text {, room } \\
\text { temperature, } 16 \mathrm{~h}\end{array}$ & $\begin{array}{l}\text { P. aeruginosa, S. aureus, B. } \\
\text { subtilis, S. paratyphi, } \\
\text { A. solani, F. oxysporum }\end{array}$ & [67] \\
\hline Atrocarpus altilis & Leaf extract & $20-50$ & Spherical & $1 \mathrm{mM}, 25^{\circ} \mathrm{C}, 24 \mathrm{~h}$ & $\begin{array}{l}\text { S. aureus, } P \text {. aeruginosa, } \\
\text { E. coli, A. vesicolor }\end{array}$ & {$[68]$} \\
\hline $\begin{array}{l}\text { Ficus benghalensis, } \\
\text { Azadirachta indica }\end{array}$ & Bark extracts & Av. 60 & Spherical & $1 \mathrm{M}, 80^{\circ} \mathrm{C}, 30 \mathrm{~min}$ & $\begin{array}{l}\text { E. coli, } P \text {. aeruginosa, } \\
\text { V. cholera, B. subtilis } \\
\text { E. coli, K. pneumonia, }\end{array}$ & {$[69]$} \\
\hline Thevetia peruviana & Leaf extract & Av. 18.1 & Spherical & $1 \mathrm{mM}, 30^{\circ} \mathrm{C}, 4 \mathrm{~h}$ & $\begin{array}{l}\text { P. aeruginosa, S. aureus, } \\
\text { B. subtilis, S. typhi }\end{array}$ & {$[70]$} \\
\hline Capparis spinosa & Leaf extract & $5-30$ & Spherical & $\begin{array}{c}10 \mathrm{mM} \text {, room } \\
\text { temperature, } 15 \mathrm{~min}\end{array}$ & $\begin{array}{l}\text { E. coli, S. typhimurium, } \\
\text { S. aureus, B. cereus }\end{array}$ & {$[71]$} \\
\hline Potentilla fulgens & Root extract & $10-15$ & Spherical & $1 \mathrm{mM}, 35^{\circ} \mathrm{C}, 18 \mathrm{~h}$ & E. coli, B. subtilis & {$[72]$} \\
\hline Petroselinum crispum & Leaf extract & $30-32$ & Spherical & $\begin{array}{c}10 \mathrm{mM} \text {, room } \\
\text { temperature, } 24 \mathrm{~h}\end{array}$ & $\begin{array}{c}\text { K. pneumoniae, E. coli, } \\
\text { S. aureus }\end{array}$ & {$[73]$} \\
\hline $\begin{array}{l}\text { Eucalyptus } \\
\text { globulus }\end{array}$ & Leaf extract & $5-25$ & Spherical, oval & $1 \mathrm{mM}, 37^{\circ} \mathrm{C}, 60 \mathrm{~min}$ & $\begin{array}{c}\text { P. aeruginosa, } \\
\text { E. coli, S. aureus }\end{array}$ & [74] \\
\hline Banana plant & Banana peel extract & 23.7 & Spherical & $1.75 \mathrm{mM}, 30^{\circ} \mathrm{C}, 72 \mathrm{~h}$ & $\begin{array}{l}\text { E. coli, P. aeruginosa, } \\
\text { B. subtilis, S. aureus }\end{array}$ & [75] \\
\hline Zingiber officinale & Rhizome & $1.4-5.7$ & Spherical & $\begin{array}{c}1 \mathrm{mM} \text {, room } \\
\text { temperature, } 1 \mathrm{~h}\end{array}$ & S. aureus, E. coli & [76] \\
\hline Erythrina indica & Root extract & $20-118$ & Spherical & $\begin{array}{l}1 \mathrm{mM} \text {, room } \\
\text { temperature, overnight }\end{array}$ & $\begin{array}{l}\text { S. aureus, M. luteus, } \\
\text { E. coli, B. subtilis, } \\
\text { S. typhi, S. paratyphi }\end{array}$ & [77] \\
\hline Prosopis farcta & Plant extract & Av. 10.8 & Spherical & $\begin{array}{c}1 \mathrm{mM} \text {, room } \\
\text { temperature, } 1 \mathrm{~h}\end{array}$ & $\begin{array}{l}\text { S. aureus, B. subtilis, } \\
\text { E. coli, P. aeruginosa }\end{array}$ & [78] \\
\hline Cassia roxburghii & Aqueous extract & $10-30$ & Spherical & $\begin{array}{c}1 \mathrm{mM} \text {, room } \\
\text { temperature, overnight }\end{array}$ & $\begin{array}{l}\text { B. subtilis, S. aureus, } \\
\text { M. luteus, P. aeruginosa, } \\
\text { E. coli, E. aerogenes }\end{array}$ & [79] \\
\hline Garcinia mangostana & Fruit extract & $30-50$ & Various & $1 \mathrm{mM}, 80^{\circ} \mathrm{C}, 15 \mathrm{~min}$ & $\begin{array}{l}\text { E. coli, P. aeruginosa, } \\
\text { S. aureus }\end{array}$ & [80] \\
\hline Panax ginseng & Root extract & $10-30$ & Spherical & $1 \mathrm{mM}, 80^{\circ} \mathrm{C}, 2 \mathrm{~h}$ & $\begin{array}{l}\text { B. anthracis, E. coli, } \\
\text { V. parahaemolyticus, } \\
\text { S. aureus, B. cereus }\end{array}$ & {$[32]$} \\
\hline Panax ginseng & Leaf extract & $5-15$ & Spherical & $1 \mathrm{mM}, 80^{\circ} \mathrm{C}, 45 \mathrm{~min}$ & $\begin{array}{c}\text { E. coli, S. enterica, } \\
\text { V. parahaemolyticus, } \\
\text { S. aureus, B. anthracis, } \\
\text { B. cereus }\end{array}$ & {$[33]$} \\
\hline $\begin{array}{c}\text { Clitoria ternatea, Solanum } \\
\text { nigrum }\end{array}$ & Leaf extract & $20-28$ & Spherical & $\begin{array}{c}100 \mathrm{mM} \text {, room } \\
\text { temperature, } \\
60 \mathrm{~min}\end{array}$ & $\begin{array}{c}\text { B. subtilis, S. aureus, } \\
\text { S. pyogenes, E. coli, } \\
\text { P. aeruginosa, K. aerogenes }\end{array}$ & [81] \\
\hline Mukia maderaspatana & Leaf extract & Av. 158 & Spherical & $\begin{array}{l}1 \mathrm{mM} \text {, room } \\
\text { temperature, } \\
15-20 \mathrm{~min}\end{array}$ & $\begin{array}{c}\text { B. subtilis, } \\
\text { K. pneumoniae, } \\
\text { S. aureus, S. typhi }\end{array}$ & [82] \\
\hline Terminalia arjuna & Plant extract & $8-16$ & Spherical & $\begin{array}{c}1 \mathrm{mM} \text {, room } \\
\text { temperature, } 15 \mathrm{~min}\end{array}$ & S. aureus, E. coli & [83] \\
\hline Eclipta alba & Leaf extract & $310-400$ & Cubic & $1 \mathrm{mM}, 32^{\circ} \mathrm{C}, 24 \mathrm{~h}$ & $\begin{array}{l}\text { E. coli, S. aureus, } \\
\text { P. aeruginosa }\end{array}$ & [84] \\
\hline Alternanthera dentata & Leaf extract & $50-100$ & Spherical & $1 \mathrm{mM}, 60^{\circ} \mathrm{C}, 45 \mathrm{~min}$ & $\begin{array}{l}\text { E. coli, P. aeruginosa, } \\
\text { K. pneumonia, E. faecalis }\end{array}$ & [85] \\
\hline Dalbergia spinosa & Leaf extract & Av. 18 & Spherical & $\begin{array}{c}100 \mathrm{mM} \text {, room } \\
\text { temperature, } 30 \mathrm{~min}\end{array}$ & $\begin{array}{l}\text { B. subtilis, } P \text {. aeruginosa, } \\
\text { S. aureus, E. coli, }\end{array}$ & [86] \\
\hline Pulicaria glutinosa & Plant extract & $40-60$ & Spherical & $1 \mathrm{mM}, 90^{\circ} \mathrm{C}, 2 \mathrm{~h}$ & $\begin{array}{l}\text { E. coli, } P \text {. aeruginosa, } \\
\text { S. aureus, M. luteus }\end{array}$ & {$[87]$} \\
\hline Phyllanthus amarus & Aqueous extract & $15.7-29.9$ & Spherical & $1 \mathrm{mM}, 70^{\circ} \mathrm{C}, 20 \mathrm{~min}$ & P. aeruginosa & [88] \\
\hline Withania somnifera & Leaf powder & $5-30$ & Spherical & $\begin{array}{c}1 \mathrm{mM} \text {, room } \\
\text { temperature, } 12 \mathrm{~h}\end{array}$ & S. aureus, E. coli & [89] \\
\hline Acorous calamus & Rhizome extract & Av. 31.8 & Spherical & $\begin{array}{l}1 \mathrm{mM} \text {, room } \\
\text { temperature, } 12 \mathrm{~h}\end{array}$ & $\begin{array}{l}\text { B. subtilis, B. cereus, } \\
\text { S. aureus }\end{array}$ & {$[90]$} \\
\hline Cocos nucifera & Plant extract & Av. 22 & Spherical & $0.9 \mathrm{mM}, 36^{\circ} \mathrm{C}, 24 \mathrm{~h}$ & $\begin{array}{l}\text { K. pneumoniae, } \\
\text { B. subtilis, } \\
\text { P. aeruginosa, } \\
\text { S. paratyphi }\end{array}$ & [91] \\
\hline Boerhaavia diffusa & Plant extract & Av. 25 & Spherical & $100 \mathrm{mM}, 24 \mathrm{~h}$ & $\begin{array}{l}\text { A. hydrophila, } \\
\text { P. fluorescens, } \\
\text { F. branchiophilum }\end{array}$ & [92] \\
\hline Azadirachta indica & Leaf extract & $4.7-18.9$ & Spherical & $\begin{array}{c}0.1 \mathrm{~N} \text {, room temperature, } \\
2 \mathrm{~h}\end{array}$ & B. subtilis, S. typhimorium & [93] \\
\hline
\end{tabular}


Table 1. Cont.

\begin{tabular}{|c|c|c|c|c|c|c|}
\hline Plants & Used Parts & Size (nm) & Shape & $\begin{array}{c}\text { Optimum Synthesis } \\
\text { Parameters }\end{array}$ & Target Pathogens & References \\
\hline Coriandrum sativum & Seed extract & $9.9-12.6$ & Spherical & $\begin{array}{l}0.1 \mathrm{~N} \text {, room temperature, } \\
2 \mathrm{~h}\end{array}$ & B. subtilis & [94] \\
\hline Hibiscus cannabinus & Leaf extract & $7-25$ & Spherical & $5 \mathrm{mM}, 30^{\circ} \mathrm{C}, 40 \mathrm{~min}$ & $\begin{array}{l}\text { E. coli, P. mirabilis, } \\
\text { S. flexneri. }\end{array}$ & [95] \\
\hline Ocimum tenuiflorum & Leaf extract & $25-40$ & NA & $\begin{array}{c}1 \mathrm{mM} \text {, room } \\
\text { temperature, } 10 \mathrm{~min}\end{array}$ & $\begin{array}{c}\text { E. coli, Corney bacterium, } \\
\text { B. substilus }\end{array}$ & [96] \\
\hline Tribulus terrestris & Fruit bodies & $16-28$ & Spherical & $\begin{array}{l}1 \mathrm{mM} \text {, room } \\
\text { temperature, } 36 \mathrm{~h}\end{array}$ & $\begin{array}{c}\text { S. pyogens, } \text { P. aeruginosa, } \\
\text { E. coli, B. subtilis, } \\
\text { S. aureus }\end{array}$ & [97] \\
\hline Lantana camara & Fruit extract & $12.5-13.0$ & Spherical & $\begin{array}{c}1 \mathrm{mM} \text {, room } \\
\text { temperature, } 1 \mathrm{~h}\end{array}$ & $\begin{array}{l}\text { M. luteus, B. subtilis, } \\
\text { S. aureus, V. cholerae, } \\
\text { K. pneumoniae, S. typhi }\end{array}$ & [98] \\
\hline Morinda citrifolia & Leaf extract & $10-60$ & Spherical & $1 \mathrm{mM}, 90^{\circ} \mathrm{C}, 60 \mathrm{~min}$ & $\begin{array}{l}\text { E. coli, P. aeroginosa, } \\
\text { K. pneumoniae, } \\
\text { E. aerogenes, B. cereus, } \\
\text { Enterococci } \mathrm{sp} .\end{array}$ & [99] \\
\hline Terminalia chebula & Plant extract & $\begin{array}{l}\text { less than } \\
100\end{array}$ & $\begin{array}{l}\text { Pentagons, } \\
\text { spherical, } \\
\text { triangular }\end{array}$ & $\begin{array}{c}2 \mathrm{mM}, \text { room } \\
\text { temperature, } 15-20 \mathrm{~min}\end{array}$ & S. aureus, E. coli & [100] \\
\hline Solanum xanthocarpum & Berry extract & $4-18$ & Spherical & $1 \mathrm{mM}, 45^{\circ} \mathrm{C}, 25 \mathrm{~min}$ & H. pylori & [101] \\
\hline Dioscorea bulbifera & Tuber extract & $8-20$ & $\begin{array}{l}\text { Nanorods, } \\
\text { triangles }\end{array}$ & $0.7 \mathrm{mM}, 50^{\circ} \mathrm{C}, 5 \mathrm{~h}$ & $\begin{array}{l}\text { E. coli, P. aeruginosa, } \\
\text { S. typhi, B. subtilis }\end{array}$ & [102] \\
\hline Garcinia mangostana & Leaf extract & Av. 35 & Spherical & $1 \mathrm{mM}, 75^{\circ} \mathrm{C}, 60 \mathrm{~min}$ & $\begin{array}{l}\text { E. coli, S. aureus } \\
\text { E.coli, S. aureus, }\end{array}$ & [103] \\
\hline Cymbopogan citratus & Leaf extract & Av. 32 & Spherical & $1 \mathrm{mM}, 37^{\circ} \mathrm{C}, 24 \mathrm{~h}$ & $\begin{array}{l}\text { P. mirabilis, S. typhi, } \\
\text { K. pnuemoniae }\end{array}$ & [104] \\
\hline Sesuvium portulacastrum L. & $\begin{array}{l}\text { Callus and leaf } \\
\text { extracts }\end{array}$ & $5-20$ & Spherical & $\begin{array}{c}1 \mathrm{mM} \text {, room } \\
\text { temperature, } 24 \mathrm{~h}\end{array}$ & $\begin{array}{l}\text { P. aeruginosa, S. aureus, } \\
\text { L. monocytogenes, } \\
\text { M. luteu, K. pneumoniae, } \\
\text { A. alternata, P. italicum, } \\
\text { F. equisetii, C. albicans }\end{array}$ & [105] \\
\hline
\end{tabular}

Av., average; NA, not available; s, second; min, minute; h, hour.

\section{Microbe Mediated Synthesis of AgNPs}

In the last few years, the potential of green synthesis of AgNPs using microorganisms has been realized (Table 2). Microorganisms have been shown to be excellent biological agents for the facile, cost effective, and ecofriendly synthesis of AgNPs, avoiding toxic and expensive chemicals and the high energy demands required for physiochemical approaches. Various microorganisms such as bacteria, yeast, fungi, and algae are often favored for the green synthesis of AgNP because of their rapid growth, simpler cultivation and ease of handling. There are two methods for the green synthesis of AgNP using microorganisms, such as the extracellular and intracellular methods [12,24]. Microorganisms synthesize various extracellular and intracellular biomolecules, including amino acid, enzymes, proteins, sugar molecules, organic materials, and many other primary and secondary metabolites [12,24]. The exact mechanism of biosynthesis of AgNP using microorganisms is still not fully known. The widely accepted mechanism of microbe-mediated synthesis of AgNPs is the chemistry of reduction and oxidation, similar to plant-mediated synthesis. First, the metal ions are reduced to NPs with the presence of microbial enzymes including reductase enzyme. Then, various extracellular and intracellular biomolecules of microorganisms serve as the capping and stabilizing agents [2,24]. Huq and Akter [106] have reported the extracellular synthesis of AgNPs from Massilia sp. MAHUQ-52. The interaction of $1 \mathrm{mM}$ $\mathrm{AgNO}_{3}$ with the bacterial culture supernatant at $30^{\circ} \mathrm{C}$ temperature yielded nanoparticles within $48 \mathrm{~h}$ of reaction. The size of synthesized AgNPs from FE-TEM analysis was found to range between 15 and $55 \mathrm{~nm}$. 
Table 2. Green synthesis of AgNPs using microorganisms and their antibacterial applications.

\begin{tabular}{|c|c|c|c|c|c|c|}
\hline Microorganisms & Method & $\begin{array}{l}\text { Size } \\
(\mathrm{nm})\end{array}$ & Shape & $\begin{array}{l}\text { Optimum Synthesis } \\
\text { Parameters }\end{array}$ & Target Pathogens & References \\
\hline Massilia sp. MAHUQ-52 & Extracellular & $15-55$ & Spherical & $1 \mathrm{mM}, 30^{\circ} \mathrm{C}, 48 \mathrm{~h}$ & $\begin{array}{l}\text { K. pneumoniae, } \\
\text { S. Enteritidis }\end{array}$ & [106] \\
\hline Streptomyces strains & Intracellular & $\begin{array}{l}1.17- \\
13.3\end{array}$ & Spherical & $5 \mathrm{mM}, 30^{\circ} \mathrm{C}, 120 \mathrm{~h}$ & $\begin{array}{c}\text { B. cereus, E. faecalis, } \\
\text { S. aureus, E. coli, S. typhi, } \\
\text { P. aeruginosa, K. pneumoniae, } P . \\
\text { vulgaris }\end{array}$ & [107] \\
\hline Cedecea sp. & Extracellular & $10-40$ & Spherical & $2 \mathrm{mM}, 37^{\circ} \mathrm{C}, 48 \mathrm{~h}$ & $\begin{array}{l}\text { E. coli, P. aeruginosa, } \\
\text { S. epidermis, S. aureus }\end{array}$ & [108] \\
\hline $\begin{array}{c}\text { Arthrobacter } \\
\text { bangladeshi }\end{array}$ & Extracellular & $12-50$ & Spherical & $1 \mathrm{mM}, 30^{\circ} \mathrm{C}, 24 \mathrm{~h}$ & S. typhimurium, Y. enterocolitica & [109] \\
\hline Aspergillus terreus & Extracellular & $60-100$ & Spherical & $100 \mathrm{mM}, 27^{\circ} \mathrm{C}, 48 \mathrm{~h}$ & $\begin{array}{c}\text { K. pneumoniae, } \\
\text { S. aureus, S. typhi, } \\
\text { P. aeruginosa, E. coli, } \\
\text { S. epidermidis, E. faecalis, } \\
\text { P. mirabilis, } \text { B. subtilis }\end{array}$ & [110] \\
\hline $\begin{array}{l}\text { Penicillium } \\
\text { chrysogenum }\end{array}$ & Extracellular & $18-60$ & Spherical & $1 \mathrm{mM}, 28^{\circ} \mathrm{C}, 24 \mathrm{~h}$ & $\begin{array}{l}\text { C. albicans, C. krusei, } \\
\text { C. tropicalis, C. parapsilosis, C. glabrata }\end{array}$ & [111] \\
\hline $\begin{array}{l}\text { Paenarthrobacter } \\
\text { nicotinovorans }\end{array}$ & Extracellular & $13-27$ & Spherical & $1 \mathrm{mM}, 30^{\circ} \mathrm{C}, 24 \mathrm{~h}$ & $\begin{array}{l}\text { B. cereus, } \\
\text { P. aeruginosa }\end{array}$ & [12] \\
\hline Aspergillus fumigatus & Intracellular & $<100$ & Spherical & $3.5 \mathrm{mM}, 25^{\circ} \mathrm{C}, 72 \mathrm{~h}$ & 11 different pathogenic bacteria & [112] \\
\hline Paenibacillus sp. & Extracellular & $\begin{array}{l}17.4- \\
52.8\end{array}$ & Spherical & $\begin{array}{l}0.1 \mathrm{mM} \text {, room } \\
\text { temperature, } 120 \mathrm{~h}\end{array}$ & $\begin{array}{l}\text { S. aureus, E. faecalis, } \\
\text { S. pneumoniae, E. coli }\end{array}$ & [113] \\
\hline Lysinibacillus xylanilyticus & Extracellular & $8-30$ & Spherical & $1 \mathrm{mM}, 30^{\circ} \mathrm{C}, 48 \mathrm{~h}$ & $\begin{array}{l}\text { V. parahaemolyticus, } \\
\text { S. Typhimurium }\end{array}$ & [114] \\
\hline $\begin{array}{l}\text { Cyanobacteria Desertifilum } \\
\text { sp. }\end{array}$ & Intracellular & $4.5-26$ & Spherical & $\begin{array}{c}1 \mathrm{mM} \text {, room } \\
\text { temperature, } 24 \mathrm{~h}\end{array}$ & $\begin{array}{c}\text { B. cereus, } \text { P. aeruginosa, B. cercus, } B . \\
\text { subtilis, } \\
\text { S. flexneri, } S \text { enterica }\end{array}$ & [115] \\
\hline Chlorella ellipsoidea & Intracellular & Av. 220 & $\begin{array}{l}\text { Spherical, cubic, } \\
\text { rod, triangular }\end{array}$ & $\begin{array}{l}1 \mathrm{mM} \text {, room } \\
\text { temperature, } 24 \mathrm{~h}\end{array}$ & $\begin{array}{l}\text { S. aureus, E. coli, } \\
\text { K. pneumoniae, } \\
\text { P. aeruginosa }\end{array}$ & [116] \\
\hline Citrobacter spp. MS5 & Extracellular & $5-15$ & Spherical & $1 \mathrm{mM}, 40{ }^{\circ} \mathrm{C}, 180 \mathrm{~min}$ & $\begin{array}{l}\text { E. hormaechei, } \\
\text { K. pneumoniae }\end{array}$ & [117] \\
\hline Sphingobium sp. MAH-11 & Extracellular & $7-22$ & Spherical & $1 \mathrm{mM}, 30^{\circ} \mathrm{C}, 48 \mathrm{~h}$ & $\begin{array}{c}\text { E. coli, S. aureus } \\
\text { S. aureus, B. subtilis, }\end{array}$ & [2] \\
\hline Padina sp. & Intracellular & $25-60$ & Spherical & $10 \mathrm{mM}, 60^{\circ} \mathrm{C}, 48 \mathrm{~h}$ & $\begin{array}{c}\text { P. aeruginosa, S. typhi, } \\
\text { E. coli }\end{array}$ & [118] \\
\hline $\begin{array}{l}\text { Chaetoceros sp., Skeletonema } \\
\text { sp., Thalassiosira sp. }\end{array}$ & Biomass & $149-239$ & $\begin{array}{l}\text { Rectangular, } \\
\text { square, regular }\end{array}$ & room temperature, $48 \mathrm{~h}$ & $\begin{array}{c}\text { E. coli, B. subtilis, } \\
\text { S. pneumonia, Aeromonas sp., } S . \\
\text { aureus }\end{array}$ & [119] \\
\hline Penicillium oxalicum & Extracellular & $60-80$ & Spherical & $1 \mathrm{mM}, 37^{\circ} \mathrm{C}, 72 \mathrm{~h}$ & $\begin{array}{c}\text { S. aureus, S. dysenteriae, } \\
\text { S. typhi }\end{array}$ & [120] \\
\hline Lactobacillus plantarum & Intracellular & Av. 14.0 & Spherical & $2 \mathrm{mM}, 37^{\circ} \mathrm{C}, 24 \mathrm{~h}$ & $\begin{array}{l}\text { S. aureus, E. coli, } \\
\text { S. epidermidis, } \\
\text { Salmonella sp. }\end{array}$ & [121] \\
\hline Escherichia coli & Extracellular & $5-50$ & Spherical & $1 \mathrm{mM}, 37^{\circ} \mathrm{C}, 72 \mathrm{~h}$ & $\begin{array}{l}\text { B. subtilis, S. aureus, } \\
\text { B. cereus, } \\
\text { P. aeruginosa, } \\
\text { K. pneumoniae, } \\
\text { E. coli, S. typhi, } \\
\text { E. vermicularis }\end{array}$ & [122] \\
\hline Terrabacter humi & Extracellular & $6-24$ & Spherical & $1 \mathrm{mM}, 30^{\circ} \mathrm{C}, 48 \mathrm{~h}$ & E. coli, P. aeruginosa & {$[20]$} \\
\hline Bacillus subtilis & Intracellular & $3-20$ & Spherical & $1 \mathrm{mM}, 30^{\circ} \mathrm{C}, 24 \mathrm{~h}$ & $\begin{array}{c}\text { S. aureus, E. coli, } \\
\text { S. epidermidis, } \\
\text { K. pneumoniae, C. albicans }\end{array}$ & [123] \\
\hline $\begin{array}{l}\text { Pseudoduganella eburnea } \\
\text { MAHUQ-39 }\end{array}$ & Extracellular & $8-24$ & Spherical & $1 \mathrm{mM}, 30^{\circ} \mathrm{C}, 24 \mathrm{~h}$ & S. aureus, $P$. aeruginosa & [6] \\
\hline $\begin{array}{c}\text { Oscillatoria } \\
\text { limnetica }\end{array}$ & Extracellular & $3.3-17.9$ & quasi-spherical & $\begin{array}{c}10 \mathrm{mM} \text {, room } \\
\text { temperature, } 48 \mathrm{~h}\end{array}$ & E. coli, B. cereus & [3] \\
\hline Acinetobacter baumannii & Extracellular & $37-168$ & Spherical & $1 \mathrm{mM}, 37^{\circ} \mathrm{C}$ & $\begin{array}{l}\text { E. coli, P. aeruginosa, } \\
\text { K. pneumoniae } \\
\text { B. cereus, S. aureus, }\end{array}$ & [124] \\
\hline $\begin{array}{l}\text { Pseudomonas sp. } \\
\text { THG-LS1.4 }\end{array}$ & Extracellular & $10-40$ & Irregular & $1 \mathrm{mM}, 28^{\circ} \mathrm{C}, 48 \mathrm{~h}$ & $\begin{array}{l}\text { C. tropicalis, } \\
\text { V. parahaemolyticus, } \\
\text { E. coli, P. aeruginosa }\end{array}$ & [125] \\
\hline $\begin{array}{l}\text { Novosphingobium sp. } \\
\text { THG-C3 }\end{array}$ & Extracellular & $8-25$ & Spherical & $1 \mathrm{mM}, 25^{\circ} \mathrm{C}, 48 \mathrm{~h}$ & $\begin{array}{l}\text { S. aureus, C. tropicalis, } \\
\text { P. aeruginosa, E. coli, } \\
\text { V. parahaemolyticus, } \\
\text { C. albicans, S. enterica, } \\
\text { B. subtilis, B. cereus }\end{array}$ & [126] \\
\hline Sporosarcina koreensis DC4 & Extracellular & $30-50$ & Spherical & $1 \mathrm{mM}, 25^{\circ} \mathrm{C}, 48 \mathrm{~h}$ & $\begin{array}{c}\text { V. parahaemolyticus, E. coli, S. enterica, } \\
\text { B. anthracis, } \\
\text { B. cereus, S. aureus }\end{array}$ & [127] \\
\hline Bacillus sp. AZ1 & Extracellular & $7-31$ & Spherical & $1 \mathrm{mM}, 40^{\circ} \mathrm{C}, 24 \mathrm{~h}$ & $\begin{array}{l}\text { S. typhi, E. coli, } \\
\text { S. epidermis, S. aureus } \\
\text { S. enterica, E. coli, } \\
\text { P. aeruoinosa. }\end{array}$ & [128] \\
\hline Aeromonas sp. THG-FG1.2 & Extracellular & $8-16$ & Spherical & $1 \mathrm{mM}, 28^{\circ} \mathrm{C}, 48 \mathrm{~h}$ & $\begin{array}{l}\text { P. aeruginosa, } \\
\text { V. parahaemolyticus, } \\
\text { B. cereus, B. subtilis, } \\
\text { S. aureus, C. albicans }\end{array}$ & [129] \\
\hline
\end{tabular}


Table 2. Cont.

\begin{tabular}{|c|c|c|c|c|c|c|}
\hline Microorganisms & Method & $\begin{array}{l}\text { Size } \\
(\mathrm{nm})\end{array}$ & Shape & $\begin{array}{c}\text { Optimum Synthesis } \\
\text { Parameters }\end{array}$ & Target Pathogens & References \\
\hline Kinneretia THG-SQI4 & Extracellular & $15-20$ & Spherical & $1 \mathrm{mM}, 28^{\circ} \mathrm{C}, 48 \mathrm{~h}$ & $\begin{array}{l}\text { C. albicans, E. coli, } \\
\text { C. tropicalis, B. cereus, } \\
\text { B. subtilis, S. aureus, } \\
\text { S. enterica, P. aeruginosa, } \\
\text { V. parahaemolyticus }\end{array}$ & [130] \\
\hline Bacillus safensis & Extracellular & $5-30$ & Spherical & $1 \mathrm{mM}, 30^{\circ} \mathrm{C}, 2 \mathrm{~h}$ & E. coli & [131] \\
\hline Aspergillus niger & Intracellular & $43-63$ & Spherical & $1 \mathrm{mM}, 35^{\circ} \mathrm{C}, 48 \mathrm{~h}$ & $\begin{array}{c}\text { K. planticola, E. coli, Pseudomonas sp., } \\
\text { B. subtilis, B. cereus } \\
\text { V. parahaemolyticus, }\end{array}$ & [132] \\
\hline Weissella oryzae & Extracellular & $10-30$ & Spherical & $1 \mathrm{mM}, 25^{\circ} \mathrm{C}, 48 \mathrm{~h}$ & $\begin{array}{l}\text { B. cereus, B. anthracis, } \\
\text { S. aureus, E. coli, } \\
\text { C. albicans }\end{array}$ & [133] \\
\hline Microbacterium resistens & Extracellular & $10-20$ & Spherical & $1 \mathrm{mM}, 30^{\circ} \mathrm{C}, 48 \mathrm{~h}$ & $\begin{array}{l}\text { S. enterica, S. aureus, } \\
\text { B. anthracis, B. cereus, } \\
\text { E. coli, C. albicans }\end{array}$ & [134] \\
\hline Bacillus methylotrophicus & Extracellular & $10-30$ & Spherical & $1 \mathrm{mM}, 28^{\circ} \mathrm{C}, 48 \mathrm{~h}$ & $\begin{array}{l}\text { V. parahaemolyticus, } \\
\text { S. enterica, E. coli, } \\
\text { C. albicans }\end{array}$ & [135] \\
\hline Pseudomonas deceptionensis & Extracellular & $10-30$ & Spherical & $1 \mathrm{mM}, 25^{\circ} \mathrm{C}, 48 \mathrm{~h}$ & $\begin{array}{l}\text { S. aureus, S. enterica, } \\
\text { V. parahaemolyticus, } \\
\text { B. anthracis, C. albicans }\end{array}$ & [136] \\
\hline Bhargavaea indica & Extracellular & $30-100$ & $\begin{array}{c}\text { Pentagon, } \\
\text { spherical, } \\
\text { hexagonal, } \\
\text { triangle, nanobar }\end{array}$ & $1 \mathrm{mM}, 25^{\circ} \mathrm{C}, 48 \mathrm{~h}$ & $\begin{array}{l}\text { V. parahaemolyticus, } \\
\text { S. enterica, S. aureus, } \\
\text { B. anthracis, B. cereus, } \\
\text { E. coli, C. albicans }\end{array}$ & [137] \\
\hline Actinomycetes & $\begin{array}{l}\text { Extracellular, } \\
\text { Intracellular }\end{array}$ & $65-80$ & Spherical & $1 \mathrm{mM}, 37^{\circ} \mathrm{C}, 72 \mathrm{~h}$ & $\begin{array}{c}\text { S. aureus, E. coli, } \\
\text { K. pneumoniae, } P \text {. vulgaris, } P \text {. } \\
\text { aeruginosa }\end{array}$ & [138] \\
\hline Bacillus flexus & Extracellular & $12-61$ & $\begin{array}{l}\text { Spherical, } \\
\text { triangular }\end{array}$ & $\begin{array}{c}1 \mathrm{mM} \text {, room } \\
\text { temperature, } 8 \mathrm{~h}\end{array}$ & $\begin{array}{l}\text { S. pyogenes, B. subtilis, } \\
\text { P. aeruginosa, E. coli }\end{array}$ & [139] \\
\hline
\end{tabular}

Singh et al. [108] have demonstrated an extracellular synthesis of AgNPs using the culture supernatant of a bacterial strain Cedecea sp. within $48 \mathrm{~h}$ of reaction and found spherical-shaped nanoparticles of 10-40 nm in size. Mondal et al. [117] have also reported the rapid synthesis of AgNPs (within $180 \mathrm{~min}$ ) using the culture supernatants of Citrobacter spp. MS5. Another report showed that AgNPs were synthesized through bioreduction of $\mathrm{AgNO}_{3}$ by the culture supernatant of Penicillium chrysogenum [111]. Sphingobium sp. MAH-11 and Pseudoduganella eburnea MAHUQ-39 have the ability to produce AgNPs with higher antibacterial activities against pathogenic microbes [2,6]. Eltarahony et al. [107] have reported the intracellular synthesis of AgNPs (within $5 \mathrm{~min}$ ) using Streptomyces strains. Hamida et al. [115] have also reported intracellular synthesis of AgNPs using Cyanobacteria Desertifilum sp. They found spherical-shaped nanoparticles of a small size, in the range of $4.5-26 \mathrm{~nm}$. Various fungi and algae were also used for facile, rapid, and ecofriendly synthesis of AgNPs. For instance, the culture supernatant of Aspergillus terreus was used to produce AgNPs with a size of 60-100 nm [110]. Raza et al. [112] have reported the intracellular synthesis of AgNPs using a fungus strain Aspergillus fumigatus KIBGE-IB33.

\section{Critical Parameters for Rapid, Facile, and Stable Synthesis of AgNPs}

Several factors play a key role for rapid, stable, and mass production of AgNPs such as the concentration of plant extracts and metal salts, incubation time, temperature, $\mathrm{pH}$, etc. (Figure 3). The shape and size of synthesized nanoparticles also depend on these factors. Extracts of the medicinal plant Potentilla fulgens was used by Mittal et al. [72] for the green synthesis of AgNPs and they found that the various physico-chemical parameters including concentrations of plant extract and metal ions, incubation time and temperature, and the $\mathrm{pH}$ of the reaction time greatly affected the rate of synthesis as well as their shape, size, and yield. They used different concentrations of plant extract (1 to $200 \mathrm{mg}$ in $50 \mathrm{~mL}$ water) and found that $4 \mathrm{mg}$ extract in $50 \mathrm{~mL}$ water was able to produce the highest concentration of AgNPs. They also used different concentrations of $\mathrm{AgNO}_{3}$ from 0.5 to $5 \mathrm{mM}$ and revealed that the yield of AgNPs increased with the increase of $\mathrm{AgNO}_{3}$ concentration from 0.5 to $1 \mathrm{mM}$, beyond which, there was again a fall in the absorbance. They found that $45^{\circ} \mathrm{C}$ is the best temperature for maximum yield and concluded that at a higher temperature, the rate of synthesis of smaller size nanoparticles increased. The 
synthesis was also influenced by the $\mathrm{pH}$ of reaction mixture. They revealed that an alkaline $\mathrm{pH}$, smaller size nanoparticles were formed, whereas at an acidic $\mathrm{pH}$, larger size nanoparticles were observed. Moreover, incubation time had a great effect on the synthesis process as well as the particle size distribution [72]. Nayak et al. [69] have reported the effect of temperature, $\mathrm{pH}$, and incubation time for the green synthesis of AgNPs using bark extracts of $A$. indica and F. benghalensis and concluded that $80^{\circ} \mathrm{C}$ temperature, a $\mathrm{pH}$ of 10 and $30 \mathrm{~min}$ incubation are the optimum conditions for rapid and stable synthesis. Similarly, Hamouda et al. [3] have shown the effect of plant extracts and $\mathrm{AgNO}_{3}$ concentrations for biosynthesis of AgNPs using an aqueous extract of Oscillatoria limnetica and reported that concentrations of the aqueous extract of Oscillatoria limnetica and $\mathrm{AgNO}_{3}$ affected the characteristics of synthesized AgNPs through controlling its size and shape. As with plant-mediated synthesis, microbe-mediated synthesis is also significantly influenced by these parameters. According to Huq [6], extracellular synthesis of AgNPs using culture supernatant of Pseudoduganella eburnea MAHUQ-39 was affected by temperature and metal salt $\left(\mathrm{AgNO}_{3}\right)$ concentration. It was found that $30{ }^{\circ} \mathrm{C}$ temperature, $1 \mathrm{mM} \mathrm{AgNO}$ (final concentration) and $24 \mathrm{~h}$ incubation time are the best conditions for the rapid and stable synthesis of AgNPs using P. eburnea. Many other recent studies also showed the effect of concentration of plant extract and metal salt, incubation time, temperature, and $\mathrm{pH}$ for the rapid and stable synthesis of homogenous AgNPs with a high yield using both plants and microbes [25,26,48,108].

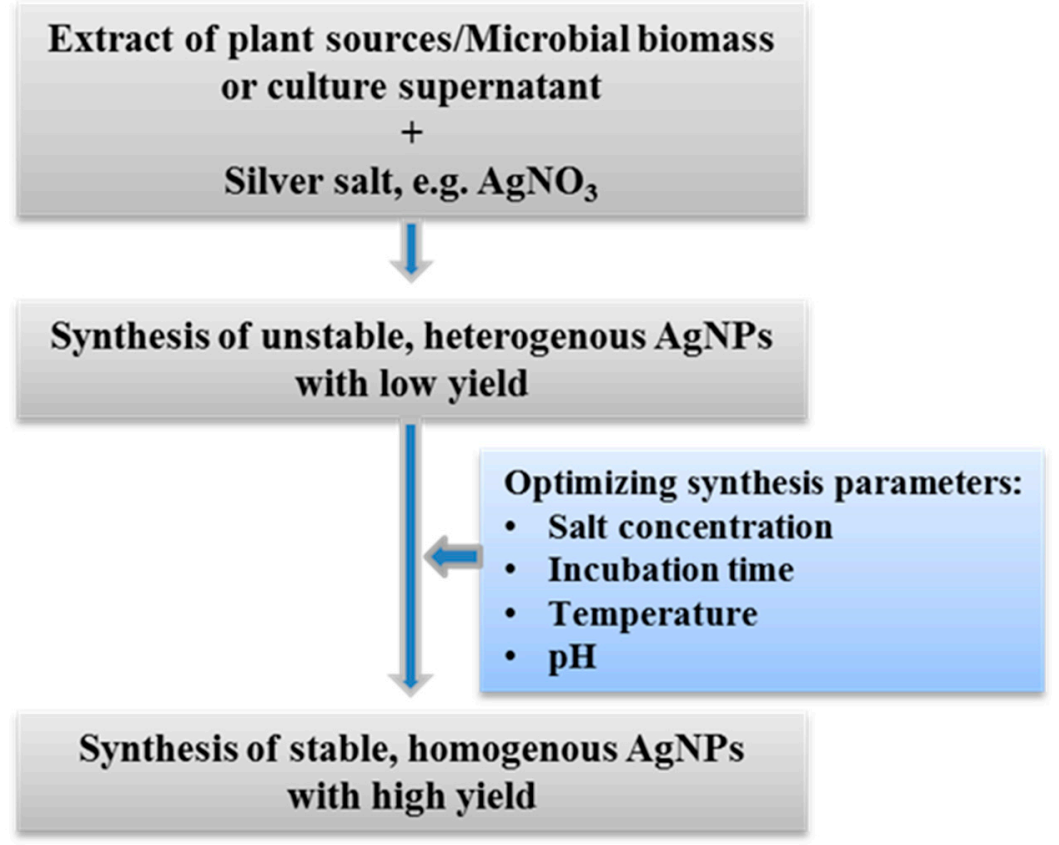

Figure 3. Optimization of parameters for stable, monodispersed, rapid and high-yield of AgNPs.

\section{Characterization of Green Synthesized AgNPs}

Characterization of AgNPs is an important step of green synthesis to check their morphology, size, shape, purity, surface chemistry, etc. Several instruments have been utilized for characterizations of green synthesized AgNPs such as UV-visible spectrophotometry, X-ray diffraction (XRD), Scanning electron microscope (SEM), Transmission electron microscope (TEM), Fourier Transform Infrared Spectroscopy (FTIR), Dynamic light scattering (DLS), and Zeta potential analyzer, etc. Synthesis of AgNPs is initially observed by the naked eye due to the change of color. Generally, the dark brown color of the reaction mixture indicates the synthesis of AgNPs. Then, the formation of AgNPs is confirmed by UV-visible spectrophotometry. Synthesized AgNPs showed a strong peak at around 400-470 nm in UV-visible spectrophotometry. The absorption spectra depended on the morphology, size and shape of biosynthesized of AgNPs [12,140]. SEM and TEM are the 
powerful tools to characterize the nanoparticles. Both SEM and TEM are used to observe the morphology, shape, size, and the degree of particle aggregation and purity of synthesized nanoparticles $[21,108,114]$. XRD is an analytical technique which has been utilized to evaluate the structural features of nanoparticles such as the degree of crystallinity, particle sizes, etc. [20]. Dynamic light scattering (DLS) is used to investigate the hydrodynamic size and polydispersity index of synthesized nanoparticles. Measurement of Zeta potential is very important to check the stability of AgNPs in aqueous suspensions. AgNPs with a Zeta potential less than $-25 \mathrm{mV}$ or greater than $+25 \mathrm{mV}$ typically have high stability [108,141].

FTIR spectroscopy is a very important tool to investigate the biomolecules responsible for the capping and stabilizing of nanoparticles [2]. Biosynthesis of AgNPs using culture supernatant of Sphingobium sp. MAH-11 and their characterization by UV-vis, TEM, XRD, DLS, and FTIR has been reported by Akter and Huq [2]. Synthesis of AgNPs was initially observed by changing of color into dark brown and finally the synthesis was confirmed on the basis of the appearance of a sharp peak at $423 \mathrm{~nm}$ in the UV-vis region of the spectrum. The TEM analysis revealed the spherical shape and the size was 7-22 nm. The SAED pattern revealed sharp rings which indicated the crystalline nature of synthesized AgNPs. The XRD pattern also showed the crystalline structure of AgNPs. The FTIR spectrum showed that various biomolecules acted as reducing agents as well as capping and stabilizing agents during the synthesis process (Figure 4), [2]. Sukweenadhi et al. [25] have reported the green synthesis of AgNPs from leaf extract of Plantago major and the synthesized AgNPs were characterized by UV-vis, TEM, SEM, XRD, DLS and FTIR.

(A)

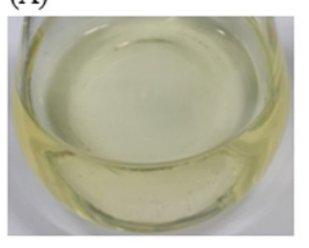

(E)

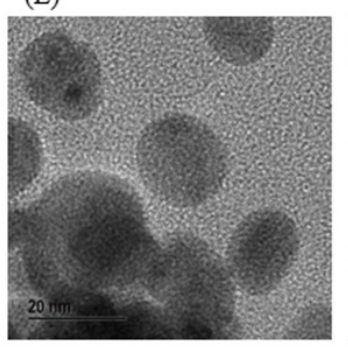

(B)

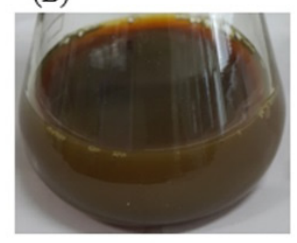

(F)

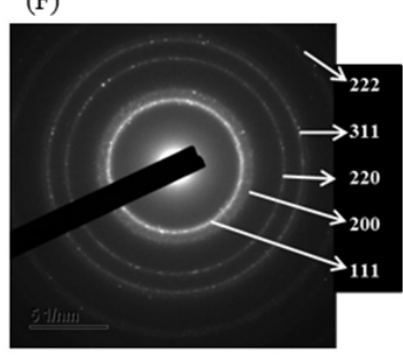

(C)

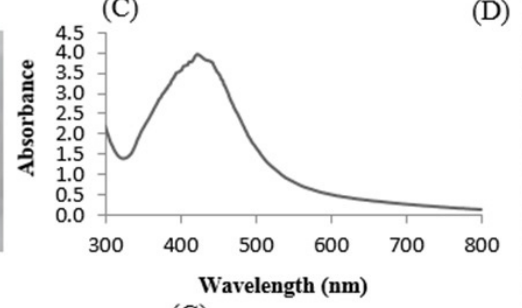

$(\mathrm{G})$
(D)

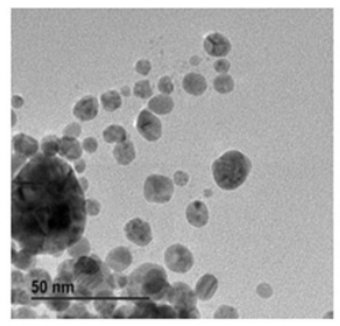

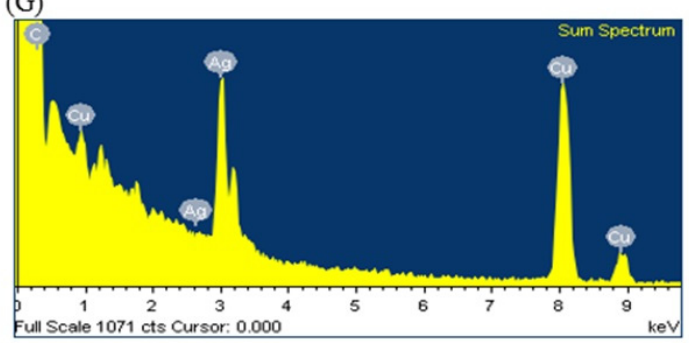

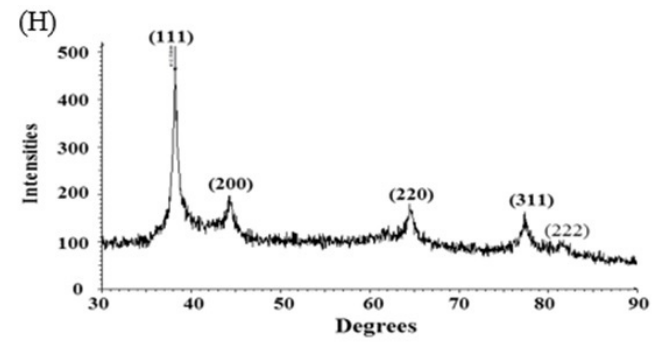

(I)

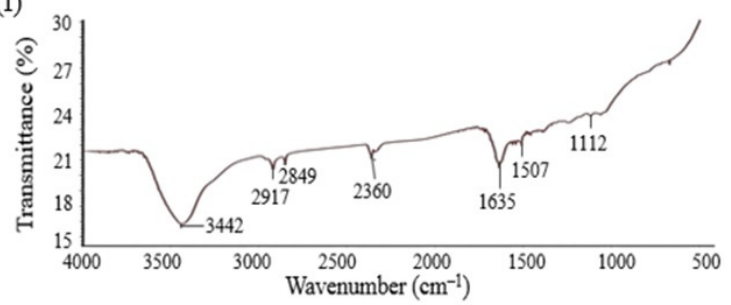

Figure 4. R2A broth with $\mathrm{AgNO}_{3}$ as control (A); biosynthesized AgNPs (B); UV-vis spectra (C); FE-TEM images (D,E); SAED pattern (F); EDX spectrum (G); X-ray diffraction pattern (H); and FT-IR spectra of biosynthesized AgNPs (I). This figure has been reprinted with permission from Ref. [2], copyright 2020, Informa UK Limited.

\section{Antibacterial Application of Green Synthesized AgNPs}

At the present time, nanoparticles have gained lots of attention by reason of the continuous improvement in treatment of bacterial infections and diseases, as well as 
inefficient treatment [142]. Among many applied nanoparticles, AgNPs have shown significant application in the reduction of pathogenic microbes and also in the treatment of microbial infections. Due to the rapid increase of antibiotic resistance in this period, this has revived the attention of the researchers investigating the therapeutic abilities of AgNPs systems as potential antimicrobial agents [142]. The published articles proposed the antibacterial activities of AgNPs, and explored them as a promising strategy which could be used as effective growth inhibitors in various microorganisms, antimicrobial control systems and for developing silver-coated medicinal devices, and silver-based dressings, such as nanogels, nanolotions, etc. [12,143,144]. The smaller particle size and greater surface volume of AgNPs holds an extensive contact area with the microbes. These features of AgNPs strongly increases their biological and chemical properties, which greatly helps them to show as robust bactericidal material [145]. This study also showed that AgNPs inhibited the growth of E. coli ATCC-15224 on both liquid as well as solid growth media. AgNPs with the concentration of $60 \mu \mathrm{g} / \mathrm{mL}$ have shown a complete cytoxicity to the E. coli bacterial strain, and the lower concentration of $60 \mu \mathrm{g} / \mathrm{mL}$ inhibited bacterial cell growth and multiplication [145].

Recently, AgNPs were synthesized using different plants and their various parts as well as bacteria, and the AgNPs produced were tested against various pathogenic microbes including multidrug-resistant bacteria (Tables 1 and 2). Huq and Akter [106] reported bacterial-mediated synthesis of AgNPs and their antibacterial activity against pathogenic strains of K. pneumoniae and S. Enteritidis. The synthesized AgNPs showed a 17.6 and a $16.8 \mathrm{~mm}$ zone of inhibition (ZOI) against K. pneumoniae and S. Enteritidis, respectively, whereas some commercial antibiotics such as erythromycin, penicillin, vancomycin, oleandomycin, novobiocin, and lincomycin were resistant or displayed very weak activity against these pathogens. The minimum inhibitory concentration (MIC)/minimum bactericidal concentration (MBC) values of synthesized AgNPs against K. pneumoniae and S. Enteritidis were 12.5/50.0 and 25.0/50.0 $\mu \mathrm{g} / \mathrm{mL}$, respectively. These MIC/MBC values were well below other antimicrobial agents including zinc oxide and gold nanoparticles against K. pneumoniae and $S$. Enteritidis. Another study reported Arthrobacter bangladeshi mediated green synthesis of AgNPs and investigated their antibacterial activity against pathogenic strains of $S$. typhimurium and Y. enterocolitica. The green synthesized AgNPs showed a 18.3 and a $20.4 \mathrm{~mm}$ ZOI against $S$. typhimurium and Y. enterocolitica, respectively. The MIC/ MBC values of synthesized AgNPs against S. typhimurium and Y. enterocolitica were $6.2 / 12.5$ and $3.1 / 12.5 \mu \mathrm{g} / \mathrm{mL}$, respectively. These MIC/MBC values were significantly lower than some other antimicrobial agents against $S$. typhimurium and Y. enterocolitica [109].

Ahmed et al. [36] reported the green synthesis of AgNPs using dried leaf extract of Tasmanian flax-lily and evaluated their antibacterial activity against several microbes including S. aureus, S. epidermidis, P. aeruginosa and C. albicans. Chlorophytum borivilianum callus extract was utilized for the green synthesis of AgNPs and the synthesized nanoparticle was used to investigate the antimicrobial activity towards the human pathogens such as B. subtilis, S. aureus, P. aeruginosa and E. coli. This result revealed that the synthesized AgNPs showed strong inhibitory activity against tested pathogens [44]. Plantago major, Prunus africana and Camellia sinensis were also reported to synthesize small-size AgNPs and evaluated against S. aureus, E. coli, P. aeruginosa and K. pneumoniae [25,35]. It has been reported that smaller size NPs showed higher antibacterial activities due to the larger surface area [146]. It was reported that AgNPs has shown remarkable antibacterial efficacy against antibiotic-resistant human pathogenic strains S. aureus, E. coli, and P. aeruginosa [2]. Hasnain et al. [45] reported on the purple heart plant leaves extract -mediated synthesis of AgNPs and evaluated their antibacterial activity against E. coli, and S. aureus. They found that the purple heart plant leaves extract-mediated synthesized AgNPs showed significantly strong antibacterial activity against both E. coli, and S. aureus compared to the purple heart plant leaves extract. Another report also proposed the excellent antimicrobial activity of biosynthesized AgNPs against various Gram-negative and Gram-positive pathogenic 
microorganisms which showed the way to use it as a potential application of antibacterial agent against multidrug-resistant bacteria [126].

Recently a few studies have stated that the conjugation of AgNPs with bactericidal agents may reduce the toxic effect towards the mammalian cells whilst increasing the bactericidal activity. This conjugation helps to increase the amount of antibacterial agent in the specific bacterial site and thus the therapeutic activity of the antibiotic agents could be enhanced against the bacterial infection $[147,148]$. It was also reported that AgNPs can be applied on a clinical platform against human pathogenic strains C. albicans, S. enterica, E. coli, and V. parahemolyticus [134,135]. It was demonstrated that green synthesis AgNPs has shown antimicrobial activity against multidrug-resistant pathogenic microbes. They also mentioned that it was ecofriendly, safe, facile, effective, and economical, which could be applied in both medical and non-medical sectors, especially as an antimicrobial agent to control drug-resistant pathogens [20]. The biosynthesized AgNPs presented great antimicrobial effect against multidrug-resistant pathogens such as $S$. aureus and P. aeruginosa. The MBCs to inhibit $S$. aureus and P. aeruginosa were 200 and $50 \mu \mathrm{g} / \mathrm{mL}$, respectively [6]. In another study this author proposed that the AgNPs synthesized by strain MAHUQ-40 showed significant antibacterial activity against V. parahaemolyticus and S. Typhimurium with MICs 3.12 and $6.25 \mu \mathrm{g} / \mathrm{mL}$, respectively [114], whereas some commercial antibiotics such as penicillin $\mathrm{G}$, erythromycin, oleandomycin, lincomycin, and vancomycin were resistant or displayed very weak activity against these pathogens. Another study investigated antimicrobial activity against both Gram-positive B. cereus and Gram-negative bacteria P. aeruginosa. The bacterial-mediated synthesized AgNPs inhibited the growth of pathogenic strains B. cereus and P. aeruginosa through developing a clear zone of inhibition [12]. Due to this killing ability, AgNPs are recognized for their remarkable antibacterial activity. Moreover, the modification in AgNPs surface developed the interactions of the constituents and this surface modification of AgNPs through chemical functionalization has gained much consideration which could be useful in numerous areas such as medical, engineering, and biological uses $[149,150]$.

\section{Antibacterial Mechanisms of AgNPs}

The most important thing about nanoparticles is their mechanism of action and this mechanism mostly depends upon the size, $\mathrm{pH}$, and ionic strength of the medium, and also on the type of capping agent. However, the complete antibacterial mechanism of AgNPs is still not fully known and has not been completely explained. According to the previous studies, it could be considered that AgNPs may frequently release the silver ions $(\mathrm{Ag}+)$, which might be considered as one of the mechanisms behind the bactericidal activity of AgNPs [142,151]. It has been demonstrated that the Ag+ ion forms complexes with the nucleic acids and interacts with the nucleosides of nucleic acids to show antibacterial activities. Nanoparticles altered the membrane permeability as evident from the release of sugars, proteins, and nuclear material through the damaged membrane [152]. The electrostatic attractions as well as an affinity towards the sulfur proteins enhanced the adhesion of Ag+ ion to the cytoplasm and cell membrane and lead to the disruptions of bacterial casings with enhancing the permeability of bacterial cell membrane [153].

The production of reactive oxygen species (ROS) is increased due to the production of free Ag+ ions by the cells, which may interrupt adenosine triphosphate (ATP) release [154]. This ROS may play an important role to disrupt the cellular membrane and the alteration in the deoxyribonucleic acid, which could cause different issues related to DNA, including DNA replication and cell propagation. On the other hand, free $\mathrm{Ag}+$ ions may efficiently interfere the protein synthesis by denaturing cytoplasmic ribosomal components [155]. The release percentage of $\mathrm{Ag}+$ ions can inhibit the growth of bacteria because the nanoscale size of AgNPs has the ability to penetrate the bacterial cell wall as well as denaturation of the cell membranes [156]. Due to denaturation of the cell membrane the intracellular and extracellular components of bacterial cell membrane may be ruptured which also causes cell lysis [157]. The antibacterial mechanisms of the AgNPs are mainly influenced 
by their dissolution profile in the reaction media and dissolution efficacy also depend on the synthesis and processing parameters [158]. Although the exact antibacterial mechanism of AgNPs has not been entirely clarified, different antibacterial actions of AgNPs have been proposed in Figure 5.

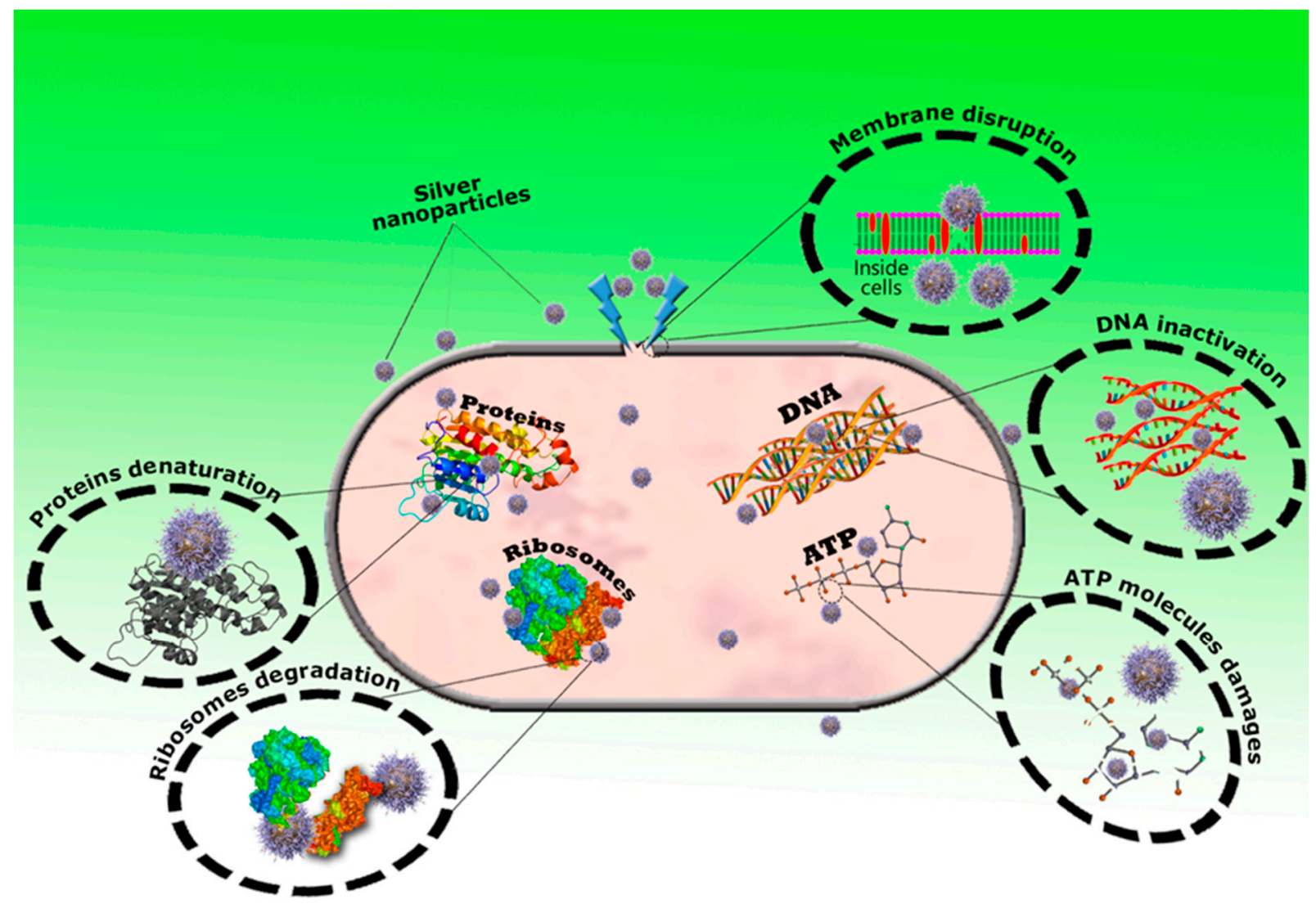

Figure 5. Possible antibacterial mechanisms of AgNPs. Disruption of cell wall and cell membrane, damage of ATP molecules due to the production of reactive oxygen species, DNA inactivation, protein denaturation and ribosome degradation.

The researchers proposed that the biosynthesized AgNPs may affect the bacterial cell morphology and penetrate the cell membrane by damaging the of cell wall of E. coli and $S$. aureus, which may decrease the reproduction of cell and ultimately lead to cell death. The FE-SEM images proved the strong antibacterial mechanism of AgNPs against pathogenic bacteria and promoted the application of AgNPs as an antimicrobial agent [2] Another study demonstrated that synthesized AgNPs changed the structural function of bacterial cells like $S$. aureus and P. aeruginosa. These mechanical activities of the proposed AgNPs create a promising hope to recognize it as an effective antimicrobial agent for various therapeutic applications against $S$. aureus and P. aeruginosa infections [6]. It was stated that AgNPs show the efficacy to alter the cell morphology as well as damage the cell membrane of tested pathogens (Figure 6). The main mechanism of AgNPs is that these nanoparticles strongly attach to the bacterial cell membrane surface and disturb its proper function, because of the enhancement of DNA damage [126]. The AgNPs also have the capability to penetrate the cell membrane and when it penetrates the cell membrane it potentially disrupts the cellular components by reacting with the sulphur-mediated proteins and phosphorus-mediated complexes like deoxyribonucleic acid [159]. Scanning electron microscopy (SEM) and TEM studies demonstrated that AgNPs shown the ability to adhere and interact with E. coli and penetrate into the bacterial cells. This adhesion and interaction ability increases the antibacterial activity of AgNPs, which are attributed with total surface area of nanoparticles [145]. Thus, the ecofriendly synthesis of AgNPs could be 
useful in various applications in both pharmaceutical and non- pharmaceutical sectors to eradicate drug-resistant pathogens [20].

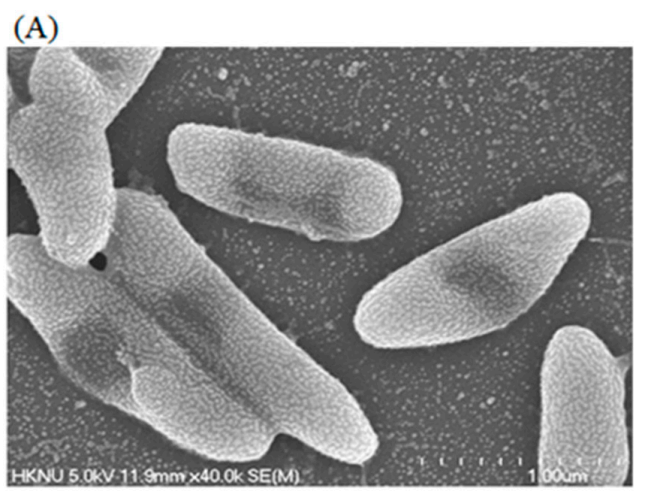

(C)

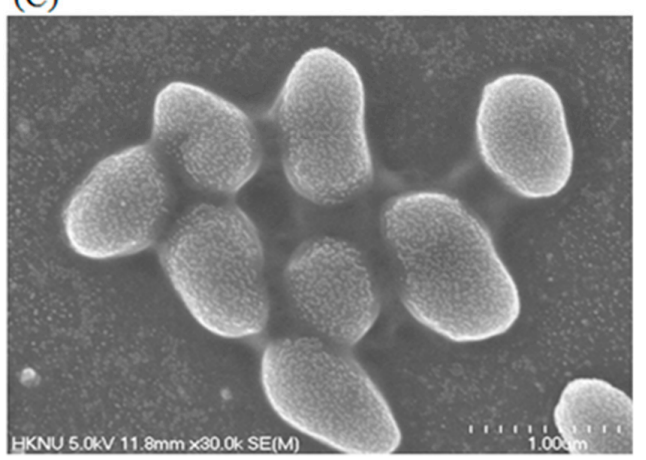

(B)

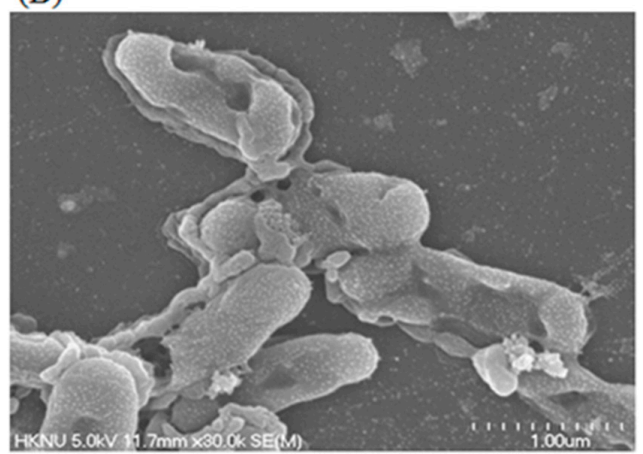

(D)

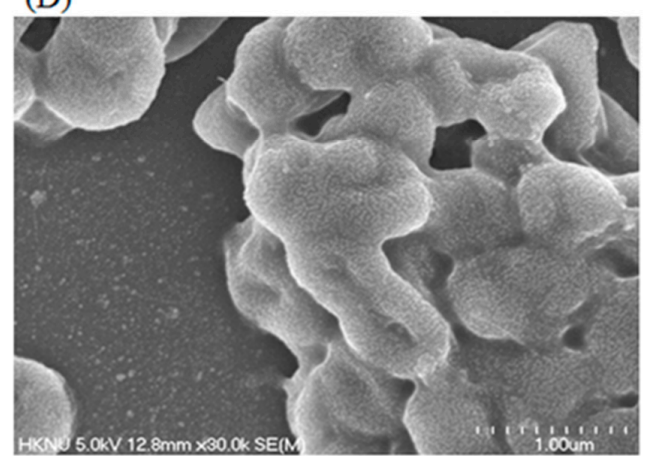

Figure 6. FE-SEM images of normal P. aeruginosa cells (A); $1 \times \mathrm{MBC}$ AgNPs treated P. aeruginosa cells (B); normal S. aureus cells (C); $1 \times$ MBC AgNPs treated S. aureus cells (D). This figure has been reprinted with permission from Ref. [6], copyright 2020, MDPI.

AgNPs have changed and damaged V. parahaemolyticus and S. typhimurium membrane integrity, which reduced the metabolic activity and normal cell function caused bacterial cells' death [114]. The field emission scanning electron microscopy analysis demonstrated that AgNPs were responsible for damaging the cell wall and altering the cell morphology of treated Gram-positive and Gram-negative pathogenic bacteria, leading to the death of cells [12]. The literature demonstrated that AgNPs trigger the inhibition of protein synthesis as well as cell wall synthesis, which provides strong evidence about the protein disruption of the outer cellular membrane and increasing ATP leakage, resulting in cell death [160].

Apart from these, the size and shape of the AgNPs increase the release of Ag+ ions owing to their greater surface area which influence potential activity against bacterial disease. The dissolution rate of AgNPs also interferes with its antimicrobial level. If the dissolution rate is high, then the potential activity could be increased [161]. It is generally proposed that AgNPs smaller than $10 \mathrm{~nm}$ may directly penetrate cell membranes, enter into the bacterial cells, and initiate cell lysis [162]. Therefore, the finding may provide a meaningful statement about AgNPs to use as an alternative antibacterial agent to protect pathogenic bacteria as well as to treat bacterial infectious diseases.

\section{Conclusions and Future Prospects}

Green synthesis of AgNPs is preferred due to its eco-friendly nature. The utilization of various parts of plant, bacteria, fungi, algae is an efficient, facile and environmentally friendly way to synthesize AgNPs. Plant extracts contain different biomolecules such as amino acids, proteins, enzymes, terpene, alkaloids, flavonoids, phenols, tannins, vitamins, etc., which act as reducing, capping, and stabilizing agents. Similarly, microorganisms synthesize various extracellular and intracellular biomolecules such as enzymes, amino acid, proteins, and many other primary and secondary metabolites that act as reducing agents as 
well as capping and stabilizing agents during the synthesis process. Biosynthesized AgNPs have great bactericidal potential against various Gram-positive and Gram-negative bacteria. In this review, green synthesis of AgNPs using plants and microbes has been comprehensively reviewed. The antibacterial applications and mechanisms of the biosynthesized AgNPs against pathogenic microbes have also been highlighted. Although the rapid, facile and eco-friendly synthetic methods using plants and microbes have shown great potential in AgNPs, the exact mechanism of synthesis and the mode of antimicrobial action are still not fully understood. Hence, several points might be considered for the future synthesis of AgNPs from plants or microbes. First, the selection of plant or microbes for easy, rapid and eco-friendly synthesis. For plant selection, researchers should consider the availability of plants and their extraction process. Plants should be available and the extraction process should be simple for facile and mass production of AgNPs. Similarly, researchers should focus on non-pathogenic and rapid growth microbes for safe and easy handling during the synthesis process. In this case, probiotic microbes could be the great synthetic agent. Second, investigation of the biomolecules present in plant extracts or in microbial biomass or culture supernatant. It is believed that different biomolecules present in plant extracts or in microbial culture supernatant are mainly responsible for the synthesis and stabilization of AgNPs. The role of various enzymes for biosynthesis needs to be studied in detail. Additionally, these biomolecules are also responsible to enhance the antibacterial efficacy of synthesized AgNPs. Therefore, it is important to investigate the biomolecules present in the plant extract or in microbial culture supernatant for successful synthesis of AgNPs. Third, optimization of parameters for rapid, stable and mass production of AgNPs. Several studies reported that various parameters such as concentration of the plant extract and $\mathrm{AgNO}_{3}$, incubation time and temperature, $\mathrm{pH}$ of reaction, etc. have great effect on synthesis process. Hence, mass production on an industrial scale can be achieved by optimizing these reaction conditions. Fourth, investigation of the antibacterial mechanisms. Most of the studies reported the efficacy of AgNPs in the screening level without investigating the exact mechanisms. It is very important to find out the mode of action of AgNPs against pathogens. Fifth, investigation of cytotoxic effect of biosynthesized AgNPs on human cells. Some studies reported that AgNPs have cytotoxic effects on human cells. Hence, it is essential to investigate the potential toxicity of biosynthesized AgNPs on healthy human cells to ensure their safe use for human and the environment.

Author Contributions: Conceptualization, M.A.H.; writing-original draft preparation, M.A.H., M.A. and S.A.; writing-review and editing, M.A.H., M.M.R. and S.R.B. All authors have read and agreed to the published version of the manuscript.

Funding: This research received no external funding.

Institutional Review Board Statement: Not applicable.

Informed Consent Statement: Not applicable.

Data Availability Statement: Not applicable.

Conflicts of Interest: The authors declare no conflict of interest.

\section{References}

1. Bachheti, R.K.; Fikadu, A.; Bachheti, A.; Husen, A. Biogenic fabrication of nanomaterials from flower-based chemical compounds, characterization and their various applications: A review. Saudi J. Biol. Sci. 2020, 27, 2551-2562. [CrossRef] [PubMed]

2. Akter, S.; Huq, M.A. Biologically rapid synthesis of silver nanoparticles by Sphingobium sp. MAH-11 T and their antibacterial activity and mechanisms investigation against drug-resistant pathogenic microbes. Artif. Cells Nanomed. Biotechnol. 2020, 48, 672-682. [CrossRef] [PubMed]

3. Hamouda, R.A.; Hussein, M.H.; Abo-Elmagd, R.A.; Bawazir, S.S. Synthesis and biological characterization of silver nanoparticles derived from the cyanobacterium Oscillatoria limnetica. Sci. Rep. 2019, 9, 13071. [CrossRef]

4. Kulkarni, N.; Muddapur, U. Biosynthesis of metal nanoparticles: A review. J. Nanotechnol. 2014, 2014, 510246. [CrossRef]

5. Jamkhande, P.G.; Ghule, N.W.; Bamer, A.H.; Kalaskar, M.G. Metal nanoparticles synthesis: An overview on methods of preparation, advantages and disadvantages, and applications. J. Drug Deliv. Sci. Technol. 2019, 53, 101174. [CrossRef] 
6. Huq, M.A. Green synthesis of silver nanoparticles using Pseudoduganella eburnea MAHUQ-39 and their antimicrobial mechanisms investigation against drug resistant human pathogens. Int. J. Mol. Sci. 2020, 21, 1510. [CrossRef]

7. Kedi, P.B.E.; Meva, F.E.; Kotsedi, L. Eco-friendly synthesis, characterization, in vitro and in vivo anti-inflammatory activity of silver nanoparticle-mediated Selaginella myosurus aqueous extract. Int. J. Nanomed. 2018, 13, 8537-8548. [CrossRef] [PubMed]

8. El-Naggar, N.E.; Hussein, M.H.; El-Sawah, A.A. Bio-fabrication of silver nanoparticles by phycocyanin, characterization, in vitro anticancer activity against breast cancer cell line and in vivo cytotoxicity. Sci. Rep. 2017, 7, 10844. [CrossRef]

9. Fouda, A.; Abdel-Maksoud, G.; Abdel-Rahman, M.A.; Eid, A.M.; Barghoth, M.G.; El-Sadany, M.A.H. Monitoring the effect of biosynthesized nanoparticles against biodeterioration of cellulose-based materials by Aspergillus niger. Cellulose 2019, 26, 6583-6597. [CrossRef]

10. Burdusel, A.C.; Gherasim, O.; Grumezescu, A.M.; Mogoanta, L.; Ficai, A.; Andronescu, E. Biomedical applications of silver nanoparticles: An up-to-date overview. Nanomaterials 2018, 8, 681. [CrossRef]

11. Rafique, M.; Sadaf, I.; Rafique, M.S.; Tahir, M.B. A review on green synthesis of silver nanoparticles and their applications. Artif. Cells Nanomed. Biotechnol. 2017, 45, 1272-1291. [CrossRef] [PubMed]

12. Huq, M.A.; Akter, S. Bacterial mediated rapid and facile synthesis of silver nanoparticles and their antimicrobial efficacy against pathogenic microorganisms. Materials 2021, 14, 2615. [CrossRef] [PubMed]

13. Salem, S.S.; Fouda, A. Green synthesis of metallic nanoparticles and their prospective biotechnological applications: An overview. Biol. Trace Elem. Res. 2021, 199, 344-370. [CrossRef] [PubMed]

14. Sharma, A.K.; Kaith, B.S.; Shanker, U.; Gupta, B. $\gamma$-radiation induced synthesis of antibacterial silver nanocomposite scaffolds derived from natural gum Boswellia serrata. J. Drug Deliv. Sci. Technol. 2020, 56, 101550. [CrossRef]

15. Elemike, E.E.; Onwudiwe, D.C.; Fayemi, O.E.; Botha, T.L. Green synthesis and electrochemistry of Ag, Au, and Ag-Au bimetallic nanoparticles using golden rod (Solidago canadensis) leaf extract. Appl. Phys. A Mater. Sci. Process. 2019, 125, 42. [CrossRef]

16. Dos Santos, M.A.; Paterno, L.G.; Moreira, S.G.C.; Sales, M.J.A. Original photochemical synthesis of Ag nanoparticles mediated by potato starch. SN Appl. Sci. 2019, 1, 554. [CrossRef]

17. Guzman, M.G.; Dille, J.; Godet, S. Synthesis of silver nanoparticles by chemical reduction method and their antibacterial activity. Int. J. Chem. Biomol. Eng. 2009, 2, 104-111.

18. Pauzi, N.; Zain, N.M.; Yusof, N.A.A. Microwave-assisted synthesis of ZnO nanoparticles stabilized with Gum Arabic: Effect of microwave irradiation time on $\mathrm{ZnO}$ nanoparticles size and morphology. Bull. Chem. React. Eng. Catal. 2019, 14, 182. [CrossRef]

19. Iravani, S.; Korbekandi, H.; Mirmohammadi, S.V.; Zolfaghari, B. Synthesis of silver nanoparticles: Chemical, physical and biological methods. Res. Pharm. Sci. 2014, 9, 385-406.

20. Akter, S.; Lee, S.-Y.; Siddiqi, M.Z.; Balusamy, S.R.; Ashrafudoulla, M.; Rupa, E.J.; Huq, M.A. Ecofriendly synthesis of silver nanoparticles by Terrabacter humi sp. nov. and their antibacterial application against antibiotic-resistant pathogens. Int. J. Mol. Sci. 2020, 21, 9746. [CrossRef]

21. Du, J.; Sing, H.; Yi, T.H. Antibacterial, anti-biofilm and anticancer potentials of green synthesized silver nanoparticles using benzoin gum (Styrax benzoin) extract. Bioprocess Biosyst. Eng. 2016, 39, 1923-1931. [CrossRef] [PubMed]

22. Vigneshwaran, N.; Kathe, A.A.; Varadarajan, P.V. Silve-protein (core-shell) nanoparticle production using spent mushroom substrate. Langmuir 2007, 23, 7113-7117. [CrossRef] [PubMed]

23. Huq, M.A. Paenibacillus anseongense sp. nov. a silver nanoparticle producing bacterium isolated from rhizospheric soil. Curr. Microbiol. 2020, 77, 2023-2030. [CrossRef] [PubMed]

24. Singh, P.; Kim, Y.J.; Zhang, D.; Yang, D.C. Biological synthesis of nanoparticles from plants and microorganisms. Trends Biotechnol. 2016, 34, 588-599. [CrossRef] [PubMed]

25. Sukweenadhi, J.; Setiawan, K.I.; Avanti, C.; Kartini, K.; Rupa, E.J.; Yang, D.C. Scale-up of green synthesis and characterization of silver nanoparticles using ethanol extract of Plantago major L. leaf and its antibacterial potential. S. Afr. J. Chem. Eng. 2021, 38, 1-8. [CrossRef]

26. Nahar, K.; Yang, D.C.; Rupa, E.J.; Khatun, M.K.; Al-Reza, S.M. Eco-friendly synthesis of silver nanoparticles from Clerodendrum viscosum leaf extract and its antibacterial potential. Nanomed. Res. J. 2020, 5, 276-287.

27. Pawar, J.S.; Patil, R.H. Green synthesis of silver nanoparticles using Eulophia herbacea (Lindl.) tuber extract and evaluation of its biological and catalytic activity. SN Appl. Sci. 2020, 2, 52. [CrossRef]

28. Soshnikova, V.; Kim, Y.J.; Singh, P.; Huo, Y.; Markus, J.; Ahn, S.; Castro-Aceituno, V.; Kang, J.; Chokkalingam, M.; Mathiyalagan, R.; et al. Cardamom fruits as a green resource for facile synthesis of gold and silver nanoparticles and their biological applications. Artif. Cells Nanomed. Biotechnol. 2018, 46, 108-117. [CrossRef]

29. Sumitha, S.; Vasanthi, S.; Shalini, S.; Chinni, S.V.; Gopinath, S.C.B.; Anbu, P.; Bahari, M.B.B.; Harish, R.; Kathiresan, S.; Ravichandran, V. Phyto-mediated photo catalysed green synthesis of silver nanoparticles using Durio Zibethinus seed extract: Antimicrobial and cytotoxic activity and photocatalytic applications. Molecules 2018, 23, 3311. [CrossRef]

30. Arokiyaraj, S.; Vincent, S.; Saravanan, M.; Lee, Y.; Oh, Y.K.; Kim, K.H. Green synthesis of silver nanoparticles using Rheum palmatum root extract and their antibacterial activity against Staphylococcus aureus and Pseudomonas aeruginosa. Artif. Cells Nanomed. Biotechnol. 2017, 45, 372-379. [CrossRef]

31. Sharma, K.; Kaushik, S.; Jyoti, A. Green Synthesis of Silver Nanoparticles by Using Waste Vegetable Peel and its Antibacterial Activities. J. Pharm. Sci. Res. 2016, 8, 313-316. 
32. Singh, P.; Kim, Y.J.; Wang, C.; Mathiyalagan, R.; Yang, D.C. The development of a green approach for the biosynthesis of silver and gold nanoparticles by using Panax ginseng root extract, and their biological applications. Artif. Cells Nanomed. Biotechnol. 2015, 44, 1150-1157. [PubMed]

33. Singh, P.; Kim, Y.J.; Yang, D.C. A strategic approach for rapid synthesis of gold and silver nanoparticles by Panax ginseng leaves. Artif. Cells Nanomed. Biotechnol. 2016, 44, 1949-1957. [CrossRef] [PubMed]

34. Adeyemi, D.K.; Adeluola, A.O.; Akinbile, M.J.; Johnson, O.O.; Ayoola, G.A. Green synthesis of Ag, Zn and Cu nanoparticles from aqueous extract of Spondias mombin leaves and evaluation of their antibacterial activity. Afr. J. Clin. Exper. Microbiol. 2020, 21, 106-113. [CrossRef]

35. Ssekatawa, K.; Byarugaba, D.K.; Kato, C.D.; Wampande, E.M.; Ejobi, F.; Nakavuma, J.L.; Maaza, M.; Sackey, J.; Nxumalo, E.; Kirabira, J.B. Green strategy-based synthesis of silver nanoparticles for antibacterial applications. Front. Nanotechnol. 2021, 3, 697303. [CrossRef]

36. Ahmed, S.R.; Anwar, H.; Ahmed, S.W.; Shah, M.R.; Ahmed, A.; Abid Ali, S.A. Green synthesis of silver nanoparticles: Antimicrobial potential and chemosensing of a mutagenic drug nitrofurazone in real samples. Measurement 2021, 180, 109489. [CrossRef]

37. Urnukhsaikhan, E.; Bold, B.E.; Gunbileg, A.; Sukhbaatar, N.; Mishig-Ochir, T. Antibacterial activity and characteristics of silver nanoparticles biosynthesized from Carduus crispus. Sci. Rep. 2021, 26, 21047. [CrossRef]

38. Aabed, K.; Mohammed, A.E. Synergistic and antagonistic effects of biogenic silver nanoparticles in combination with antibiotics against some pathogenic microbes. Front. Bioeng. Biotechnol. 2021, 9, 249. [CrossRef]

39. Madivoli, E.S.; Kareru, P.G.; Gachanja, A.N.; Mugo, S.M.; Makhanu, D.S.; Wanakai, S.I.; Gavamukulya, Y. Facile synthesis of silver nanoparticles using Lantana trifolia aqueous extracts and their antibacterial activity. J. Inorg. Organomet. Polym. 2020, 30, 2842-2850. [CrossRef]

40. Chavan, R.R.; Bhinge, S.D.; Bhutkar, M.A.; Randive, D.S.; Wadkar, G.H.; Todkar, S.S. Characterization, antioxidant, antimicrobial and cytotoxic activities of green synthesized silver and iron nanoparticles using alcoholic Blumea eriantha DC plant extract Urade. Mater. Today Commun. 2020, 24, 101320. [CrossRef]

41. Hemlata; Meena, P.R.; Singh, A.P.; Tejavath, K.K. Biosynthesis of silver nanoparticles using Cucumis prophetarum aqueous leaf extract and their antibacterial and antiproliferative activity against cancer cell lines. ACS Omega 2020, 5, 5520-5528. [CrossRef]

42. Shejawal, K.P.; Randive, D.S.; Bhinge, S.D.; Bhutkar, M.A.; Wadkar, G.H.; Jadhav, N.R. Green synthesis of silver and iron nanoparticles of isolated proanthocyanidin: Its characterization, antioxidant, antimicrobial, and cytotoxic activities against COLO320DM and HT. J. Genet. Eng. Biotechnol. 2020, 18, 43. [CrossRef] [PubMed]

43. Kalpana, D.; Han, J.H.; Park, W.S.; Lee, S.M.; Wahab, R.; Lee, Y.S. Green biosynthesis of silver nanoparticles using Torreya nucifera and their antibacterial activity. Arab. J. Chem. 2019, 12, 1722-1732. [CrossRef]

44. Huang, F.; Long, Y.; Liang, Q.; Purushotham, B.; Swamy, M.K.; Duan, Y. Safed musli (Chlorophytum borivilianum L.) callus-mediated biosynthesis of silver nanoparticles and evaluation of their antimicrobial activity and cytotoxicity against human colon cancer cells. J. Nanomater. 2019, 2019, 2418785. [CrossRef]

45. Hasnain, M.S.; Javed, N.; Alam, S.; Rishishwar, P.; Rishishwar, S.; Ali, S.; Nayak, A.K.; Beg, S. Purple heart plant leaves extract-mediated silver nanoparticle synthesis: Optimization by Box-Behnken design. Mater. Sci. Eng. C 2019, 99, 1105-1114. [CrossRef]

46. Oves, M.; Aslam, M.; Rauf, M.A.; Qayyum, S.; Qari, H.A.; Khan, M.S. Antimicrobial and anticancer activities of silver nanoparticles synthesized from the root hair extract of Phoenix dactylifera. Mater. Sci. Eng. C 2018, 89, 429-443. [CrossRef] [PubMed]

47. Saratale, R.G.; Benelli, G.; Kumar, G.; Kim, D.S.; Saratale, G.D. Bio-Fabrication of silver nanoparticles using the leaf extract of an ancient herbal medicine, dandelion (Taraxacum officinale), evaluation of their antioxidant, anticancer potential, and antimicrobial activity against phytopathogens. Environ. Sci. Pollut. Res. 2017, 25, 10392-10406. [CrossRef] [PubMed]

48. Khatami, M.; Zafarnia, N.; Heydarpoor Bami, M.; Sharifi, I.; Singh, H. Antifungal and antibacterial activity of densely dispersed silver nanospheres with homogeneity size which synthesized using chicory: An in vitro study. J. Mycol. Med. 2018, 28, 637-644. [CrossRef]

49. Devanesan, S.; AlSalhi, M.S.; Balaji, R.V.; Ranjitsingh, A.J.A.; Ahamed, A.; Alfuraydi, A.A.; AlQahtani, F.Y.; Aleanizy, F.S.; Othman, A.H. Antimicrobial and cytotoxicity effects of synthesized silver nanoparticles from Punica granatum peel extract. Nanoscale Res. Lett. 2018, 13, 315. [CrossRef]

50. Mythili, R.; Selvankumar, T.; Kamala-Kannan, S.; Sudhakar, C.; Ameen, F.; Al-Sabri, A.; Selvam, K.; Govarthanan, M.; Kim, H. Utilization of market vegetable waste for silver nanoparticle synthesis and its antibacterial activity. Mater. Lett. 2018, 225, 101-104. [CrossRef]

51. Markus, J.; Wang, D.; Kim, Y.J.; Ahn, S.; Mathiyalagan, R.; Wang, C.; Yang, D.C. Biosynthesis, characterization, and bioactivities evaluation of silver and gold nanoparticles mediated by the roots of Chinese Herbal Angelica pubescens maxim. Nanoscale Res. Lett. 2017, 12, 46. [CrossRef] [PubMed]

52. Mohanta, Y.K.; Panda, S.K.; Bastia, A.K.; Mohanta, T.K. Biosynthesis of silver nanoparticles from Protium serratum and investigation of their potential impacts on food safety and control. Front. Microbiol. 2017, 8, 626. [CrossRef]

53. Huo, Y.; Singh, P.; Kim, Y.J.; Soshnikova, V.; Kang, J.; Markus, J.; Ahn, S.; Castro-Aceituno, V.; Mathiyalagan, R.; Chokkalingam, M.; et al. Biological synthesis of gold and silver chloride nanoparticles by Glycyrrhiza uralensis and in vitro applications. Artif. Cells Nanomed. Biotechnol. 2018, 46, 303-312. [CrossRef] [PubMed] 
54. Nasar, S.; Murtaza, G.; Mehmood, A.; Bhatti, T.M. Green approach to synthesis of silver nanoparticles using Ficus Palmata leaf extract and their antibacterial profile. Pharm. Chem. J. 2017, 51, 811-817. [CrossRef]

55. Rajkuberan, C.; Prabukumar, S.; Sathishkumar, G.; Wilson, A.; Ravindran, K.; Sivaramakrishnan, S. Facile synthesis of silver nanoparticles using Euphorbia antiquorum L. latex extract and evaluation of their biomedical perspectives as anticancer agents. J. Saudi Chem. Soc. 2017, 21, 911-919. [CrossRef]

56. Jain, S.; Mehata, M.S. Medicinal plant leaf extract and pure flavonoid mediated green synthesis of silver nanoparticles and their enhanced antibacterial property. Sci. Rep. 2017, 7, 15867. [CrossRef]

57. Mitiku, A.A.; Yilma, B. Antibacterial and antioxidant activity of silver nanoparticles synthesized using aqueous extract of Moringa stenopetala leaves. Afr. J. Biotechnol. 2017, 16, 1705-1716.

58. Singh, H.; Du, J.; Singh, P.; Yi, T.H. Ecofriendly synthesis of silver and gold nanoparticles by Euphrasia officinalis leaf extract and its biomedical applications. Artif. Cells Nanomed. Biotechnol. 2017, 46, 1163-1170. [CrossRef]

59. Abbai, R.; Ramya Mathiyalagan, J.M.; Kim, Y.J.; Wang, C.; Singh, P.; Ahn, S.; Farh, M.E.A.; Yang, D.C. Green synthesis of multifunctional silver and gold nanoparticles from the oriental herbal adaptogen: Siberian ginseng. Int. J. Nanomed. 2016, 11, 3131-3143.

60. Singh, H.; Du, J.; Yi, T.H. Green and rapid synthesis of silver nanoparticles using Borago officinalis leaf extract: Anticancer and antibacterial activities. Artif. Cells Nanomed. Biotechnol. 2017, 45, 1310-1316. [CrossRef]

61. Lateef, A.; Azeez, M.A.; Asafa, T.B.; Yekeen, T.A.; Akinboro, A.; Oladipo, I.C.; Azeez, L.; Ojo, S.A.; Gueguim-Kana, E.B.; Beukes, L.S. Cocoa pod husk extract-mediated biosynthesis of silver nanoparticles: Its antimicrobial, antioxidant and larvicidal activities. J. Nanostruct. Chem. 2016, 6, 159-169. [CrossRef]

62. Ahmed, S.; Saifullah; Ahmad, M.; Swami, B.L.; Ikram, S. Green synthesis of silver nanoparticles using Azadirachta indica aqueous leaf extract. J. Radiat. Res. Appl. Sci. 2016, 9, 1-7. [CrossRef]

63. Anandalakshmi, K.; Venugobal, J.; Ramasamy, V. Characterization of silver nanoparticles by green synthesis method using Pedalium murex leaf extract and their antibacterial activity. Appl. Nanosci. 2016, 6, 399-408. [CrossRef]

64. Mohanta, Y.K.; Panda, S.K.; Biswas, K.; Tamang, A.; Bandyopadhyay, J.; Bastia, A.K. Biogenic synthesis of silver nanoparticles from Cassia fistula (Linn.): In vitro assessment of their antioxidant, antimicrobial and cytotoxic activities. IET Nanobiotechnol. 2016, 10, 438-444. [CrossRef] [PubMed]

65. Bose, D.; Chatterjee, S. Biogenic synthesis of silver nanoparticles using guava (Psidium guajava) leaf extract and its antibacterial activity against Pseudomonas aeruginosa. Appl. Nanosci. 2016, 6, 895-901. [CrossRef]

66. Dhand, V.; Soumya, L.; Bharadwaj, S.; Chakra, S.; Bhatt, D.; Sreedhar, B. Green synthesis of silver nanoparticles using Coffea arabica seed extract and its antibacterial activity. Mater. Sci. Eng. C 2016, 58, 36-43. [CrossRef]

67. Sundararajan, B.; Mahendran, G.; Thamaraiselvi, R.; Kumari, B.D. Biological activities of synthesized silver nanoparticles from Cardiospermum halicacabum L. Bull. Mater. Sci. 2016, 39, 423-431.

68. Ravichandran, V.; Vasanthi, S.; Shalini, S.; Ali-Shah, S.A.; Harish, R. Green synthesis of silver nanoparticles using Atrocarpus altilis leaf extract and the study of their antimicrobial and antioxidant activity. Mater. Lett. 2016, 180, 264-267. [CrossRef]

69. Nayak, D.; Ashe, S.; Rauta, P.R.; Kumari, M.; Nayak, B. Bark extract mediated green synthesis of silver nanoparticles: Evaluation of antimicrobial activity and antiproliferative response against osteosarcoma. Mater. Sci. Eng. C 2016, 58, 44-52. [CrossRef]

70. Oluwaniyi, O.O.; Adegoke, H.I.; Adesuji, E.T.; Alabi, A.B.; Bodede, S.O.; Labulo, A.H.; Oseghale, C.O. Biosynthesis of silver nanoparticles using aqueous leaf extract of Thevetia peruviana Juss and its antimicrobial activities. Appl. Nanosci. 2016, 6, 903-912. [CrossRef]

71. Benakashania, F.; Allafchian, A.R.; Jalali, S.A.H. Biosynthesis of silver nanoparticles using Capparis spinosa L. leaf extract and their antibacterial activity. Karbala Int. J. Mod. Sci. 2016, 2, 251-258. [CrossRef]

72. Mittal, A.K.; Tripathy, D.; Choudhary, A.; Aili, P.K.; Chatterjee, A.; Singh, I.P.; Banerjee, U.C. Bio-synthesis of silver nanoparticles using potentilla fulgens wall. ex Hook. and its therapeutic evaluation as anticancer and antimicrobial agent. Mater. Sci. Eng. C 2015, 53, 120-127. [CrossRef] [PubMed]

73. Roy, K.; Sarkar, C.; Ghosh, C. Plant-mediated synthesis of silver nanoparticles using parsley (Petroselinum crispum) leaf extract: Spectral analysis of the particles and antibacterial study. Appl. Nanosci. 2015, 5, 945-951. [CrossRef]

74. Ali, K.; Ahmed, B.; Dwivedi, S.; Saquib, Q.; Al-Khedhairy, A.A.; Musarrat, J. Microwave accelerated green synthesis of stable silver nanoparticles with Eucalyptus globulus leaf extract and their antibacterial and antibiofilm activity on clinical isolates. PLoS ONE 2015, 10, e0131178. [CrossRef]

75. Ibrahim, H.M. Green synthesis and characterization of silver nanoparticles using banana peel extract and their antimicrobial activity against representative microorganisms. J. Radiat. Res. Appl. Sc. 2015, 8, 265-275. [CrossRef]

76. Shalaby, T.I.; Mahmoud, O.A.; Batouti, G.A.E.; Ibrahim, E.E. Green synthesis of silver nanoparticles: Synthesis, characterization and antibacterial activity. Nanosci. Nanotechnol. 2015, 5, 23-29.

77. Sre, P.R.; Reka, M.; Poovazhagi, R.; Kumar, M.A.; Murugesan, K. Antibacterial and cytotoxic effect of biologically synthesized silver nanoparticles using aqueous root extract of Erythrina indica lam. Spectrochim. Acta Part A Mol. Biomol. Spectrosc. 2015, 135, 1137-1144.

78. Miri, A.; Sarani, M.; Bazaz, M.R.; Darroudi, M. Plant-mediated biosynthesis of silver nanoparticles using Prosopis farcta extract and its antibacterial properties. Spectrochim. Acta A Mol. Biomol. Spectrosc. 2015, 141, 287-291. [CrossRef] 
79. Balashanmugam, P.; Kalaichelvan, P.T. Biosynthesis characterization of silver nanoparticles using Cassia roxburghii DC. aqueous extract, and coated on cotton cloth for effective antibacterial activity. Int. J. Nanomed. 2015, 10, 87. [CrossRef]

80. Rajakannu, S.; Shankar, S.; Perumal, S.; Subramanian, S.; Dhakshinamoorthy, G.P. Biosynthesis of silver nanoparticles using Garcinia mangostana fruit extract and their antibacterial, antioxidant activity. Int. J. Curr. Microbiol. Appl. Sci. 2015, 4, 944-952.

81. Krithiga, N.; Rajalakshmi, A.; Jayachitra, A. Green synthesis of silver nanoparticles using leaf extracts of Clitoria ternatea and Solanum nigrum and study of its antibacterial effect against common nosocomial pathogens. J. Nanosci. 2015, 8, 928204. [CrossRef]

82. Harshiny, M.; Matheswaran, M.; Arthanareeswaran, G.; Kumaran, S.; Rajasree, S. Enhancement of antibacterial properties of silver nanoparticles-ceftriaxone conjugate through Mukia maderaspatana leaf extract mediated synthesis. Ecotoxicol. Environ. Saf. 2015, 121, 135-141. [CrossRef] [PubMed]

83. Ahmed, S.; Ikram, S. Silver nanoparticles: One pot green synthesis using Terminalia arjuna extract for biological application. Nano Res. Appl. 2015, 1, 309.

84. Premasudha, P.; Venkataramana, M.; Abirami, M.; Vanathi, P.; Krishna, K.; Rajendran, R. Biological synthesis and characterization of silver nanoparticles using Eclipta alba leaf extract and evaluation of its cytotoxic and antimicrobial Potential. Bull. Mater. Sci. 2015, 38, 965-973. [CrossRef]

85. Kumar, D.A.; Palanichamy, V.; Roopan, S.M. Green synthesis of silver nanoparticles using Alternanthera dentata leaf extract at room temperature and their antimicrobial activity. Spectrochim. Acta A Mol. Biomol. Spectrosc. 2014, 127, 168-171. [CrossRef]

86. Muniyappan, N.; Nagarajan, N.S. Green synthesis of silver nanoparticles with Dalbergia spinosa leaves and their applications in biological and catalytic activities. Process Biochem. 2014, 49, 1054-1061. [CrossRef]

87. Khan, M.; Khan, S.T.; Khan, M.; Adil, S.F.; Musarrat, J.; Al-Khedhairy, A.A.; Al-Warthan, A.; Siddiqui, M.; Alkhathlan, H.Z. Antibacterial properties of silver nanoparticles synthesized using Pulicaria glutinosa plant extract as a green bioreductant. Int. J. Nanomed. 2014, 9, 3551-3565.

88. Singh, K.; Panghal, M.; Kadyan, S.; Chaudhary, U.; Yadav, J.P. Green silver nanoparticles of Phyllanthus amarus: As an antibacterial agent against multi drug resistant clinical isolates of Pseudomonas aeruginosa. J. Nanobiotechnol. 2014, 12, 40. [CrossRef]

89. Raut, R.W.; Mendhulkar, V.D.; Kashid, S.B. Photosensitized synthesis of silver nanoparticles using Withania somnifera leaf powder and silver nitrate, J. Photochem. Photobiol. B 2014, 132, 45-55. [CrossRef]

90. Nakkala, J.R.; Mata, R.; Gupta, A.K.; Sadras, S.R. Biological activities of green silver nanoparticles synthesized with Acorous calamus rhizome extract, Eur. J. Med. Chem. 2014, 85, 784-794. [CrossRef]

91. Mariselvam, R.; Ranjitsingh, A.; Nanthini, A.U.R.; Kalirajan, K.; Padmalatha, C.; Selvakumar, P.M. Green synthesis of silver nanoparticles from the extract of the inflorescence of Cocos nucifera (Family: Arecaceae) for enhanced antibacterial activity. Spectrochim. Acta Part A Mol. Biomol. Spectrosc. 2014, 129, 537-541. [CrossRef] [PubMed]

92. Kumar, P.V.; Pammi, S.; Kollu, P.; Satyanarayana, K.; Shameem, U. Green synthesis and characterization of silver nanoparticles using Boerhaavia diffusa plant extract and their anti bacterial activity. Ind. Crops Prod. 2014, 52, 562-566. [CrossRef]

93. Nazeruddin, G.M.; Prasad, N.R.; Waghmare, S.R.; Garadkar, K.M.; Mulla, I.S. Extracellular biosynthesis of silver nanoparticle using Azadirachta indica leaf extract and its anti-microbial activity. J. Alloy. Compd. 2014, 583, 272-277. [CrossRef]

94. Nazeruddin, G.M.; Prasad, N.R.; Prasadd, S.R.; Shaikha, Y.I.; Waghmare, S.R.; Adhyapak, P. Coriandrum sativum seed extract assisted in situ green synthesis ofsilver nanoparticle and its anti-microbial activity. Ind. Crops Prod. 2014, 60, 212-216. [CrossRef]

95. Bindhu, M.R.; Umadevi, M. Synthesis of monodispersed silver nanoparticles using Hibiscus cannabinus leaf extract and its antimicrobial activity. Spectrochim. Acta A Mol. Biomol. Spectrosc. 2013, 101, 184-190. [CrossRef]

96. Patil, R.S.; Kokate, M.R.; Kolekar, S.S. Bioinspired synthesis of highly stabilized silver nanoparticles using Ocimum tenuiflorum leaf extract and their antibacterial activity. Spectrochim. Acta A 2012, 91, 234-238. [CrossRef]

97. Gopinath, V.; MubarakAli, D.; Priyadarshini, S.; Priyadharsshini, N.M.; Thajuddin, N.; Velusamy, P. Biosynthesis of silver nanoparticles from Tribulus terrestris and its antimicrobial activity: A novel biological approach. Colloids Surf. B Biointerfaces 2012, 96, 69-74. [CrossRef]

98. Sivakumar, P.; Nethradevi, C.; Renganathan, S. Synthesis of silver nanoparticles using Lantana camara fruit extract and its effect on pathogens, Asian J. Pharm. Clin. Res. 2012, 5, 97-101.

99. Sathishkumar, G.; Gobinatha, C.; Karpagama, K.; Hemamalini, V.; Premkumar, K.; Sivaramakrishnan, S. Phyto-synthesis of silver nanoscale particles using Morinda citrifolia L. and its inhibitory activity against human pathogens. Colloids Surf. B Biointerfaces 2012, 95, 235-240. [CrossRef]

100. Kumar, K.M.; Sinha, M.; Mandal, B.K.; Ghosh, A.R.; Siva Kumar, K.; Sreedhara Reddy, P. Green synthesis of silver nanoparticles using Terminalia chebula extract at room temperature and their antimicrobial studies. Spectrochim. Acta A Mol. Biomol. Spectrosc. 2012, 91, 228-233. [CrossRef]

101. Amin, M.; Anwar, F.; Janjua, M.R.S.A.; Iqbal, M.A.; Rashid, U. Green Synthesis of Silver Nanoparticles through Reduction with Solanum xanthocarpum L. Berry Extract: Characterization, Antimicrobial and Urease Inhibitory Activities against Helicobacter pylori. Int. J. Mol. Sci. 2012, 13, 9923-9941. [CrossRef] [PubMed]

102. Ghosh, S.; Patil, S.; Ahire, M.; Kitture, R.; Jabgunde, A.; Kale, S.; Pardesi, K.; Cameotra, S.S.; Bellare, J.; Dhavale, D.D.; et al. Synthesis of silver nanoparticles using Dioscorea bulbifera tuber extract and evaluation of its synergistic potential in combination with antimicrobial agents. Int. J. Nanomed. 2012, 7, 483-496. 
103. Veerasamy, R.; Xin, T.Z.; Gunasagaran, S.; Xiang, T.F.W.; Yang, E.F.C.; Jeyakumar, N.; Dhanaraj, S.A. Biosynthesis of silver nanoparticles using mangosteen leaf extract and evaluation of their antimicrobial activities, J. Saudi Chem. Society. 2011, 15, 113-120. [CrossRef]

104. Masurkar, S.A.; Chaudhari, P.R.; Shidore, V.B.; Kamble, S.P. Rapid biosynthesis of silver nanoparticles using Cymbopogan Citratus (Lemongrass) and its antimicrobial activity. Nano-Micro Lett. 2011, 3, 189-194. [CrossRef]

105. Nabikhan, A.; Kandasamy, K.; Raj, A.; Alikunhi, N.M. Synthesis of antimicrobial silver nanoparticles by callus and leaf extracts from saltmarsh plant, Sesuvium portulacastrum L. Colloids Surf. B 2010, 79, 488-493. [CrossRef]

106. Huq, M.A.; Akter, S. Biosynthesis, characterization and antibacterial application of novel silver nanoparticles against drug resistant pathogenic Klebsiella pneumoniae and Salmonella enteritidis. Molecules 2021, 26, 5996. [CrossRef] [PubMed]

107. Eltarahony, M.; Ibrahim, A.; El-shall, H.; Ibrahim, E.; Althobaiti, F.; Fayad, E. Antibacterial, antifungal and antibiofilm activities of silver nanoparticles supported by crude bioactive metabolites of bionanofactories isolated from lake mariout. Molecules 2021, 26, 3027. [CrossRef]

108. Singh, P.; Pandit, S.; Jers, C.; Abhayraj, S.; Garnæs, J.; Mijakovic, I. Silver nanoparticles produced from Cedecea sp. exhibit antibiofilm activity and remarkable stability. Sci. Rep. 2021, 11, 12619. [CrossRef]

109. Huq, M.A.; Akter, S. Characterization and genome analysis of Arthrobacter bangladeshi sp. nov., applied for the green synthesis of silver nanoparticles and their antibacterial efficacy against drug-resistant human pathogens. Pharmaceutics 2021, $13,1691$. [CrossRef]

110. Vellingiri, M.M.; Ashwin, J.K.M.; Soundari, A.J.P.G.; Sathiskumar, S.; Priyadharshini, U.; Paramasivam, D.; Liu, W.C.; Balasubramanian, B. Mycofabrication of AgONPs derived from Aspergillus terreus FC36AY1 and its potent antimicrobial, antioxidant, and anti-angiogenesis activities. Mol. Biol. Rep. 2021, 48, 7933-7946. [CrossRef]

111. Soliman, A.M.; Abdel-Latif, W.; Shehata, I.H.; Fouda, A.; Abdo, A.M.; Ahmed, Y.M. Green approach to overcome the resistance pattern of Candida spp. using biosynthesized silver nanoparticles fabricated by Penicillium chrysogenum F9. Biol. Trace Elem. Res. 2021, 199, 800-811. [CrossRef] [PubMed]

112. Raza, S.; Ansari, A.; Siddiqui, N.N.; Ibrahim, F.; Abro, M.I.; Aman, A. Biosynthesis of silver nanoparticles for the fabrication of non cytotoxic and antibacterial metallic polymer based nanocomposite system. Sci. Rep. 2021, 11, 10500. [CrossRef] [PubMed]

113. Sreenivasa, N.; Meghashyama, B.P.; Pallavi, S.S.; Bidhayak, C.; Dattatraya, A.; Muthuraj, R.; Shashiraj, K.N.; Halaswamy, H.; Dhanyakumara, S.B.; Vaishnavi, M.D. Biogenic synthesis of silver nanoparticles using Paenibacillus sp. in-vitro and their antibacterial, anticancer activity assessment against human colon tumour cell line. J. Environ. Biol. 2021, 42, 118-127. [CrossRef]

114. Huq, M.A. Biogenic silver nanoparticles synthesized by Lysinibacillus xylanilyticus MAHUQ-40 to control antibiotic-resistant human pathogens vibrio parahaemolyticus and salmonella typhimurium. Front. Bioeng. Biotechnol. 2020, 8, 1407. [CrossRef] [PubMed]

115. Hamida, R.S.; Abdelmeguid, N.E.; Ali, M.A.; Bin-Meferij, M.M.; Khalil, M.I. Synthesis of silver nanoparticles using a novel cyanobacteria Desertifilum sp. extract: Their antibacterial and cytotoxicity effects. Int. J. Nanomed. 2020, 15, 49-63. [CrossRef]

116. Borah, D.; Das, N.; Das, N.; Bhattacharjee, A.; Sarmah, P.; Ghosh, K.; Chandel, M.; Rout, J.; Pandey, P.; Ghosh, N.N.; et al. Alga-mediated facile green synthesis of silver nanoparticles: Photophysical, catalytic and antibacterial activity. Appl. Organ. Chem. 2020, 34, e5597. [CrossRef]

117. Mondal, A.H.; Yadav, D.; Ali, A.; Khan, N.; Jin, J.O.; Haq, Q.M.R. Anti-bacterial and anti-candidal activity of silver nanoparticles biosynthesized using Citrobacter spp. Ms5 culture supernatant. Biomolecules 2020, 10, 944. [CrossRef]

118. Bhuyar, P.; Rahim, M.H.A.; Sundararaju, S.; Ramaraj, R.; Maniam, G.P.; Govindan, N. Synthesis of silver nanoparticles using marine macroalgae Padina sp. and its antibacterial activity towards pathogenic bacteria. Beni-Suef Univ. J. Basic Appl. Sci. 2020, 9, 3. [CrossRef]

119. Mishra, B.; Saxena, A.; Tiwari, A. Biosynthesis of silver nanoparticles from marine diatoms Chaetoceros sp., Skeletonema sp., Thalassiosira sp., and their antibacterial study. Biotechnol. Rep. 2020, 28, e00571. [CrossRef] [PubMed]

120. Feroze, N.; Arshad, B.; Younas, M.; Afridi, M.I.; Saqib, S.; Ayaz, A. Fungal mediated synthesis of silver nanoparticles and evaluation of antibacterial activity. Microsc. Res. Tech. 2020, 83, 72-80. [CrossRef]

121. Mohd-Yusof, H.; Rahman, A.; Mohamad, R.; Zaidan, U.H. Microbial mediated synthesis of silver nanoparticles by Lactobacillus Plantarum TA4 and its antibacterial and antioxidant activity. Appl. Sci. 2020, 10, 6973. [CrossRef]

122. Saeed, S.; Iqbal, A.; Ashraf, M.A. Bacterial-mediated synthesis of silver nanoparticles and their significant effect against pathogens. Environ. Sci. Pollut. Res. 2020, 27, 37347-37356. [CrossRef] [PubMed]

123. Alsamhary, K.I. Eco-friendly synthesis of silver nanoparticles by Bacillus subtilis and their antibacterial activity. Saudi J. Biol. Sci. 2020, 27, 2185-2191. [CrossRef] [PubMed]

124. Shaker, M.A.; Shaaban, M.I. Synthesis of silver nanoparticles with antimicrobial and anti-adherence activities against multidrugresistant isolates from Acinetobacter Baumannii. J. Taibah Univ. Med. Sci. 2017, 12, 291-297. [CrossRef]

125. Singh, H.; Du, J.; Singh, P.; Yi, T.H. Extracellular synthesis of silver nanoparticles by Pseudomonas sp. THG-LS1.4 and their antimicrobial application. J. Pharm. Anal. 2018, 8, 258-264. [CrossRef]

126. Du, J.; Sing, H.; Yi, T.H. Biosynthesis of silver nanoparticles by Novosphingobium sp. THG-C3 and their antimicrobial potential. Artif. Cells Nanomed. Biotechnol. 2017, 45, 211-217. [CrossRef]

127. Singh, P.; Singh, H.; Kim, Y.J. Extracellular synthesis of silver and gold nanoparticles by Sporosarcina koreensis DC4 and their biological applications. Enzym. Microb. Technol. 2016, 86, 75-83. [CrossRef] 
128. Deljou, A.; Goudarzi, S. Green extracellular synthesis of the silver nanoparticles using thermophilic Bacillus Sp. AZ1 and its antimicrobial activity against several human pathogenetic bacteria. Iran. J. Biotechnol. 2016, 14, 25-32. [CrossRef]

129. Singh, H.; Du, J.; Yi, T.H. Biosynthesis of silver nanoparticles using Aeromonas sp. THG-FG1.2 and its antibacterial activity against pathogenic microbes. Artif. Cells Nanomed. Biotechnol. 2017, 45, 584-590. [CrossRef]

130. Singh, H.; Du, J.; Yi, T.H. Kinneretia THG-SQI4 mediated biosynthesis of silver nanoparticles and its antimicrobial efficacy. Artif. Cells Nanomed. Biotechnol. 2017, 45, 602-608. [CrossRef]

131. Lateef, A.; Adelere, I.A.; Gueguim-Kana, E.B.; Asafa, T.B.; Beukes, L.S. Green synthesis of silver nanoparticles using keratinase obtained from a strain of Bacillus safensis LAU 13. Int. Nano Lett. 2015, 5, 29-35. [CrossRef]

132. Rajeshkumar, S.; Paulkumar, K.; Gnanajobitha, G.; Chitra, K.; Malarkodi, C.; Annadurai, G. Fungal assisted intracellular and enzyme based synthesis of silver nanoparticles and its bactericidal efficiency. Int. Res. J. Pharm. Biosci. 2015, 2, 8-19.

133. Singh, P.; Kim, Y.J.; Wang, C.; Mathiyalagan, R.; Yang, D.C. Weissella oryzae DC6-facilitated green synthesis of silver nanoparticles and their antimicrobial potential. Artif. Cells Nanomed. Biotechnol. 2015, 44, 1569-1575. [CrossRef] [PubMed]

134. Wang, C.; Singh, P.; Kim, Y.J.; Mathiyalagan, R.; Myagmarjav, D.; Wang, D.; Jin, C.-G.; Yang, D.C. Characterization and antimicrobial application of biosynthesized gold and silver nanoparticles by using Microbacterium resistens. Artif. Cells Nanomed. Biotechnol. 2016, 44, 1714-1721. [CrossRef] [PubMed]

135. Wang, C.; Kim, Y.J.; Singh, P.; Mathiyalagan, R.; Jin, Y.; Yang, D.C. Green synthesis of silver nanoparticles by Bacillus methylotrophicus, and their antimicrobial activity. Artif. Cells Nanomed. Biotechnol. 2015, 44, 1127-1132. [PubMed]

136. Jo, J.H.; Singh, P.; Kim, Y.J.; Wang, C.; Mathiyalagan, R.; Jin, C.-G.; Yang, D.C. Pseudomonas deceptionensis DC5-mediated synthesis of extracellular silver nanoparticles. Artif. Cells Nanomed. Biotechnol. 2015, 44, 1576-1581. [CrossRef]

137. Singh, P.; Kim, Y.J.; Singh, H.; Mathiyalagan, R.; Wang, C.; Yang, D.C. Biosynthesis of anisotropic silver nanoparticles by Bhargavaea indica and their synergistic effect with antibiotics against pathogenic microorganisms. J. Nanomater. 2015, 2, 4.

138. Abdeen, S.; Geo, S.; Praseetha, P.K.; Dhanya, R.P. Biosynthesis of silver nanoparticles from Actinomycetes for therapeutic applications. Int. J. Nano Dimen. 2014, 5, 155-162.

139. Priyadarshini, S.; Gopinath, V.; Priyadharsshini, N.M.; Mubarak, A.D.; Velusamy, P. Synthesis of anisotropic silver nanoparticles using novel strain, Bacillus flexus and its biomedical application. Coll. Surf. B Biointerface 2013, 102, 232-237. [CrossRef]

140. Tomaszewska, E.; Soliwoda, K.; Kadziola, K.; Tkacz-Szczesna, B.; Celichowski, G.; Cichomski, M.; Szmaja, W.; Grobelny, J. Detection limits of DLS and UV-Vis spectroscopy in characterization of polydisperse nanoparticles colloids. J. Nanomater. 2013, 2013, 313081. [CrossRef]

141. Rajeshkumar, S.; Bharath, L.V. Mechanism of plant-mediated synthesis of silver nanoparticles e A review on biomolecules involved, characterisation and antibacterial activity. Chem.-Biol. Interact. 2017, 273, 219-227. [CrossRef] [PubMed]

142. Anees-Ahmad, S.; Sachi-Das, S.; Khatoon, A.; Ansari, M.T.; Afzal, M.; Saquib-Hasnain, M.; Kumar-Nayak, A. Bactericidal activity of silver nanoparticles: A mechanistic review. Mater. Sci. Energy Technol. 2020, 3, 756-769. [CrossRef]

143. Mahendra, R.; Yadav, A.; Gade, A. Silver nanoparticles as a new generation of antimicrobials. Biotechnol. Adv. $2009,27,76-83$.

144. Kim, J.S.; Kuk, E.; Yu, K.N.; Kim, J.-H.; Park, S.J.; Lee, H.J.; Kim, S.H.; Park, Y.K.; Park, Y.H.; Hwang, C.-Y.; et al. Antimicrobial effects of silver nanoparticles. Nanomed. Nanotechnol. Biol. Med. 2007, 3, 95-101. [CrossRef] [PubMed]

145. Raffi, M.; Hussain, F.; Bhatti, T.M.; Akhter, J.I.; Hameed, A.; Hasan, M.M. Antibacterial characterization of silver nanoparticles against E. coli ATCC-15224. J. Mater. Sci. Technol. 2008, 24, 192-196.

146. Goswami, S.R.; Sahareen, T.; Singh, M.; Kumar, S. Role of biogenic silver nanoparticles in disruption of cell-cell adhesion in Staphylococcus aureus and Escherichia coli biofilm. J. Ind. Eng. Chem. 2015, 26, 73-80. [CrossRef]

147. Allahverdiyev, A.M.; Kon, K.V.; Abamor, E.S.; Bagirova, M.; Rafailovich, M. Coping with antibiotic resistance: Combining nanoparticles with antibiotics and other antimicrobial agents. Expert Rev. Anti. Infect. Ther. 2011, 9, 1035-1052. [CrossRef]

148. Sekhon, B.S. Metalloantibiotics and antibiotic mimics-an overview. J. Pharm. Educ. Res. 2010, 1, 1.

149. Das, S.S.; Neelam; Hussain, K.S.; Singh, S.; Hussain, A.; Faruk, A.; Tebyetekerwa, M. Laponite-based nanomaterials for biomedical applications: A review. Curr. Pharm. Des. 2019, 25, 424-443. [CrossRef]

150. Shetti, N.P.; Nayak, D.S.; Malode, S.J.; Reddy, K.R.; Shukla, S.S.; Aminabhavi, T.M. Electrochemical behavior of flufenamic acid at amberlite XAD-4 resin and silver-doped titanium dioxide/amberlite XAD-4 resin modified carbon electrodes. Colloids Surf. B Biointerfaces 2019, 177, 407-415. [CrossRef]

151. Bapat, R.A.; Chaubal, T.V.; Joshi, C.P.; Bapat, P.R.; Choudhury, H.; Pandey, M.; Gorain, B.; Kesharwani, P. An overview of application of silver nanoparticles for biomaterials in dentistry. Mater. Sci. Eng. C 2018, 91, 881-898. [CrossRef] [PubMed]

152. Chouhan, S.; Guleria, S. Green synthesis of AgNPs using Cannabis sativa leaf extract: Characterization, antibacterial, anti-yeast and $\alpha$-amylase inhibitory activity. Mater. Sci. Energy Technol. 2020, 3, 536-544. [CrossRef]

153. Khorrami, S.; Zarrabi, A.; Khaleghi, M.; Danaei, M.; Mozafari, M. Selective cytotoxicity of green synthesized silver nanoparticles against the MCF-7 tumor cell line and their enhanced antioxidant and antimicrobial properties. Int. J. Nanomed. 2018, 13, 8013-8024. [CrossRef] [PubMed]

154. Das, S.S.; Alkahtani, S.; Bharadwaj, P.; Ansari, M.T.; ALKahtani, M.D.F.; Pang, Z.; Hasnain, M.S.; Nayak, A.K.; Aminabhavi, T.M. Molecular insights and novel approaches for targeting tumor metastasis. Int. J. Pharm. 2020, 585, 119556. [CrossRef]

155. Pareek, V.; Gupta, R.; Panwar, J. Do physico-chemical properties of silver nanoparticles decide their interaction with biological media and bactericidal action? A Review. Mater. Sci. Eng. C Mater. Biol. Appl. 2018, 90, 739-749. [CrossRef] [PubMed] 
156. Liao, C.; Li, Y.; Tjong, S.C. Bactericidal and cytotoxic properties of silver nanoparticles. Int. J. Mol. Sci. 2019, 20, 449. [CrossRef] [PubMed]

157. Ahmed, K.B.R.; Nagy, A.M.; Brown, R.P.; Zhang, Q.; Malghan, S.G.; Goering, P.L. Silver nanoparticles: Significance of physicochemical properties and assay interference on the interpretation of in vitro cytotoxicity studies. Toxicol. Vitr. 2017, 38, 179-192. [CrossRef] [PubMed]

158. Noronha, V.T.; Paula, A.J.; Duran, G.; Galembeck, A.; Cogo-Mueller, K.; Franz-Montan, M.; Duran, N. Silver nanoparticles in dentistry. Dent. Mater. 2017, 33, 1110-1126. [CrossRef]

159. Soumya, E.A.; Saad, I.K.; Hassan, L.; Ghizlane, Z.; Hind, M.; Adnane, R. Carvacrol and thymol components inhibiting Pseudomonas aeruginosa adherence and biofilm formation. Afr. J. Microbiol. Res. 2011, 5, 3229-3232.

160. Park, J.; Lim, D.H.; Lim, H.J.; Kwon, T.; Choi, J.S.; Jeong, S.; Choi, I.H.; Cheon, J. Size dependent macrophage responses and toxicological effects of Ag nanoparticles. Chem. Commun. 2011, 47, 4382-4384. [CrossRef]

161. Jacob, J.M.; John, M.S.; Jacob, A.; Abitha, P.; Kumar, S.S.; Rajan, R.; Natarajan, S.; Pugazhendhi, A. Bactericidal coating of paper towels via sustainable biosynthesis of silver nanoparticles using Ocimum sanctum leaf extract. Mater. Res. Express $2019,6,45401$. [CrossRef]

162. Saravanan, M.; Arokiyaraj, S.; Lakshmi, T.; Pugazhendhi, A. Synthesis of silver nanoparticles from Phenerochaete chrysosporium (MTCC-787) and their antibacterial activity Antibacterial Activity against Human Pathogenic Bacteria. Microb. Pathog. 2018, 117, 68-72. [CrossRef] [PubMed] 